\title{
BOUNDED HOMOTOPY EQUIVALENCES OF HILBERT CUBE MANIFOLDS
}

BY

\author{
C. BRUCE HUGHES
}

\begin{abstract}
ABSTRaCr. Let $M$ and $F$ be Hilbert cube manifolds with $F$ compact. The purpose of this paper is to study homotopy equivalences $f: M \rightarrow \mathbf{R}^{m} \times F$ which have bounded control in the $\mathbf{R}^{m}$-direction. Roughly, these homotopy equivalences form a semi-simplicial complex $\mathscr{W} h\left(\mathbf{R}^{m} \times F\right)$, the controlled Whitehead space. Using results about approximate fibrations, $\mathscr{W} h\left(\mathbf{R}^{m} \times F\right)$ is related to the semi-simplicial complex of bounded concordances on $\mathbf{R}^{m} \times F$. Then the homotopy groups of $\mathscr{W} h\left(\mathbf{R}^{m} \times F\right)$ are computed in terms of the lower algebraic $K$-theoretic functors $K_{-i}$.
\end{abstract}

1. Introduction. Let $F$ be a compact Hilbert cube manifold. We are interested in homotopy equivalences $f: M \rightarrow \mathbf{R}^{m} \times F$ which are controlled in the $\mathbf{R}^{m}$-direction, where $M$ is also a Hilbert cube manifold. To say $f$ is controlled in the $\mathbf{R}^{m}$-direction means that $p f: M \rightarrow \mathbf{R}^{m}$ is an approximate fibration, where $p: \mathbf{R}^{m} \times F \rightarrow \mathbf{R}^{m}$ is projection. The collection of all such homotopy equivalences, which are additionally given to be retractions onto the collared submanifold $\mathbf{R}^{m} \times F$ of $M$, form the vertices of a semi-simplicial complex $\mathscr{W} h\left(\mathbf{R}^{m} \times F\right)$ (see $\$ 2$ for the precise definition).

The main result of this paper is the computation of the homotopy groups of $\mathscr{W} h\left(\mathbf{R}^{m} \times F\right)$ (see Corollary 1 below). In order to do this we relate $\mathscr{W} h\left(\mathbf{R}^{m} \times F\right)$ to the semi-simplicial complex $\mathscr{C}_{b}\left(\mathbf{R}^{m} \times F\right)$ of bounded concordances on $\mathbf{R}^{m} \times F$. An $n$-simplex of $\mathscr{C}_{b}\left(\mathbf{R}^{m} \times F\right)$ is a homeomorphism

$$
h: \mathbf{R}^{m} \times F \times[0,1] \times \Delta^{n} \rightarrow \mathbf{R}^{m} \times F \times[0,1] \times \Delta^{n}
$$

such that $h$ is fiber preserving over $\Delta^{n}, h \mid\left(\mathbf{R}^{m} \times F \times\{0\} \times \Delta^{n}\right) \cup\left(\mathbf{R}^{m} \times F \times[0,1] \times\right.$ $\partial \Delta^{n}$ ) is the identity, and $h$ is bounded (that is, there is a constant $L>0$ such that $p h$ is $L$-close to $p$, where $p$ is projection to $\mathbf{R}^{m}$ ). We can now state our first result.

THEOREM 1. There is a group isomorphism $\alpha: \pi_{n} \mathscr{W} h\left(\mathbf{R}^{m} \times F\right) \rightarrow \pi_{n-1} \mathscr{C}_{b}\left(\mathbf{R}^{m} \times F\right)$ for each $m \geqslant 0, n \geqslant 1$.

The proof of this theorem (which is given in \$5), is based on a sharpened version of the main result of [24] which shows how to straighten out certain parameterized families of approximate fibrations to be nearly like a product family. This sharpened version is Theorem 2.3 in $\S 2$.

Received by the editors January 17, 1983 and, in revised form, March 24, 1983.

1980 Mathematics Subject Classification. Primary 57N20; Secondary 55R65.

Key words and phrases. Approximate fibration, bounded homotopy equivalence, concordance, Hilbert cube manifold, lower algebraic $K$-theory. 
The next result illustrates a further relationship between $\mathscr{W} h\left(\mathbf{R}^{m} \times F\right)$ and $\mathscr{C}_{b}\left(\mathbf{R}^{m} \times F\right)$.

THEOREM 2. There is a group isomorphism $\beta: \pi_{n} \mathscr{C}_{b}\left(\mathbf{R}^{m+1} \times F\right) \rightarrow \pi_{n} \mathscr{W} h\left(\mathbf{R}^{m} \times F\right)$ for each $m \geqslant 0, n \geqslant 0$.

The proof of this theorem (which is given in §6) is based on a familiar method for finding a Whitehead torsion invariant of a bounded concordance on $M \times \mathbf{R}$ (see [1, 29 and 30]). In fact, the proof of Theorem 2 is motivated by [1].

Together, Theorems 1 and 2 reduce the study of $\pi_{n} \mathscr{W} h\left(\mathbf{R}^{m} \times F\right)$ for $m \geqslant n$ to the study of $\pi_{0} \mathscr{W} h\left(\mathbf{R}^{m-n} \times F\right)$. In $\$ 4$ we reinterpret a result of Chapman [8] to obtain the following

THEOREM 3. There is a group isomorphism

$$
\pi_{0} \mathscr{W} h\left(\mathbf{R}^{m} \times F\right) \approx \begin{cases}K_{1-m}\left(\mathbf{Z} \pi_{1}(F)\right) & \text { if } m>1, \\ \tilde{K}_{0}\left(\mathbf{Z} \pi_{1}(F)\right) & \text { if } m=1, \\ \operatorname{Wh}\left(\mathbf{Z} \pi_{1}(F)\right) & \text { if } m=0 .\end{cases}
$$

Here Wh denotes the usual algebraically defined Whitehead group functor, $\tilde{K}_{0}$ is the reduced projective class group functor, and $K_{-i}(i>0)$ denotes the lower algebraic $K$-theoretic functor of Bass [2] (see also Gersten [19]). As usual, $\mathbf{Z} \pi_{1}(F)$ is the integral group ring of the fundamental group of $F$. For calculations of $K_{-i}$ see [3, 4, and 5].

Combining Theorems 1, 2 and 3 we get the following corollary which is the main result of this paper.

COROLLARY 1. There is a group isomorphism

$$
\pi_{n} \mathscr{W} h\left(\mathbf{R}^{m} \times F\right) \approx \begin{cases}K_{1-m+n}\left(\mathbf{Z} \pi_{1}(F)\right) & \text { if } 0 \leqslant n \leqslant m-2, \\ \tilde{K}_{0}\left(\mathbf{Z} \pi_{1}(F)\right) & \text { if } n=m-1, \\ \operatorname{Wh}\left(\mathbf{Z} \pi_{1}(F)\right) & \text { if } n=m, \\ \pi_{n-m-1} \mathscr{C}(F) & \text { if } n>m .\end{cases}
$$

Here $\mathscr{C}(F)$ denotes the semi-simplicial complex of concordances on $F$. In addition, we also get the following expected Hilbert cube manifold version of the Anderson-Hsiang result on bounded concordances [1, Theorem 3].

COROLlARY 2. There is a group isomorphism

$$
\pi_{n} \mathscr{C}_{b}\left(\mathbf{R}^{m} \times F\right) \approx \begin{cases}K_{2-m+n}\left(\mathbf{Z} \pi_{1}(F)\right) & \text { if } 0 \leqslant n \leqslant m-3, \\ \tilde{K}_{0}\left(\mathbf{Z} \pi_{1}(F)\right) & \text { if } n=m-2, \\ \operatorname{Wh}\left(\mathbf{Z} \pi_{1}(F)\right) & \text { if } n=m-1, \\ \pi_{n-m} \mathscr{C}(F) & \text { if } n \geqslant m .\end{cases}
$$

In [7 and 8] Chapman has studied controlled homotopy equivalences $f: M \rightarrow E$ from a Hilbert cube manifold $M$ into the total space $E$ of a locally trivial fiber bundle $p: E \rightarrow B$ with compact Hilbert cube manifold fiber $F$ and polyhedral base 
B. (See related work for finite-dimensional manifolds by Chapman [9] and Quinn $[27,28]$.) The main problem is to decide when $f$ can be approximated arbitrarily closely by a homeomorphism (with the closeness measured in the base $B$ ). Theorem 3 of [8] says $f$ can be so approximated provided $\pi_{1}(F)$ is "nice enough." It is hoped that the results of this paper on controlled homotopy equivalences into the trivial bundle $p: \mathbf{R}^{m} \times F \rightarrow \mathbf{R}^{m}$ can be used to obtain a general answer to this problem.

This paper is organized as follows. $\S 2$ contains the definition of $\mathscr{W} h\left(\mathbf{R}^{m} \times F\right)$, general preliminaries and the result on parameterized approximate fibrations mentioned above. $\$ 3$ gives a geometric understanding of the homotopy relation in $\mathscr{W} h\left(\mathbf{R}^{m} \times F\right)$. Also in $\S 3$, we define the torsion, for certain $n$-parameter families of homotopy equivalences to $\mathbf{R}^{m} \times F$ with only bounded control in the $\mathbf{R}^{m}$-direction, to be an element of $\pi_{n} \mathscr{W} h\left(\mathbf{R}^{m} \times F\right)$. In $\S 4$ we discuss the group structure of $\pi_{0} \mathscr{W} h\left(\mathbf{R}^{m} \times F\right)$ and prove Theorem 3. The proofs of Theorems 1 and 2 are given in $\S \S 5$ and 6, respectively.

The author wishes to express his gratitude to T. A. Chapman for suggesting to him that the main theorem of [24] could be used to obtain the results of this paper.

2. Preliminaries. In this section the spaces $\mathscr{W} h\left(\mathbf{R}^{m} \times F\right)$ are defined. The key property of parametrized families of approximation fibrations needed for the constructions in the sequel is established in Theorem 2.3.

We begin with some notation. The Hilbert cube is denoted by $Q$ and a Hilbert cube manifold or $Q$-manifold is a separable metric space which is locally homeomorphic to $Q$. The reader should consult [6] for the basic machinery of $Q$-manifolds including the notion of $Z$-sets. Throughout this paper $F$ will denote a compact $Q$-manifold, $\mathbf{R}^{m}$ euclidean $m$-space, and $\Delta^{n}$ the standard $n$-simplex with combinatorial boundary $\partial \Delta^{n}$ consisting of the faces $\partial_{0} \Delta^{n}, \partial_{1} \Delta^{n}, \ldots, \partial_{n} \Delta^{n}$. If $m$ or $n$ is 0 , then $\mathbf{R}^{m}$ or $\Delta^{n}$ will denote a point.

We will often encounter fiber preserving (f.p.) maps and almost always these will be maps which preserve the obvious fibers over $\Delta^{n}$. Specifically, if $\rho: X \rightarrow \Delta^{n}, \sigma$ : $Y \rightarrow \Delta^{n}$ and $f: X \rightarrow Y$ are maps, then $f$ is f.p. if $\sigma f=\rho$. Often the maps $\rho$ and $\sigma$ will be understood to be some natural projections. The map $p$ will almost always denote projection to $\mathbf{R}^{m}$ or $\mathbf{R}^{m} \times \Delta^{n}$, depending on the context. The space $\mathbf{R}^{m}$ is given the metric induced by the norm $\|x\|=\max \left\{\left|x_{1}\right|, \ldots,\left|x_{m}\right|\right\}$. For fiber preserving, or sliced, $Q$-manifold results (including the notion of sliced $Z$-sets) see [12 and 13].

A map is proper if the inverse image of every compact set is compact. To say a map is a bundle means that the map is the projection map of a locally trivial fiber bundle.

The maps which will make up the space $\mathscr{W} h\left(\mathbf{R}^{m} \times F\right)$ are defined as follows. Let $\rho: M \rightarrow \Delta^{n}$ be a bundle with $Q$-manifold fiber such that $\mathbf{R}^{m} \times F \times \Delta^{n}$ is a closed subset of $M$ and the inclusion $\mathbf{R}^{m} \times F \times \Delta^{n} \rightarrow M$ is f.p. If $\varepsilon>0$ and $f: M \rightarrow \mathbf{R}^{m} \times$ $F \times \Delta^{n}$ is an f.p. proper retraction, then $f$ is said to be an $f \cdot p \cdot p^{-1}(\varepsilon)$-sdr provided there exists an f.p. homotopy $F: M \times[0,1] \rightarrow M$ such that $F_{0}=\mathrm{id}, F_{1}=f, F_{t} \mid \mathbf{R}^{m} \times$ $F \times \Delta^{n}=$ id for $0 \leqslant t \leqslant 1$, and the diameter of $p f\{F(\{x\} \times[0,1])\}$ is less than $\varepsilon$ for each $x$ in $M$. If $f$ is an f.p. $p^{-1}(\varepsilon)$-sdr for some (possibly large) $\varepsilon>0$, then $f$ is an $f$.p. bounded sdr. 
The space $\mathscr{W} h\left(\mathbf{R}^{m} \times F\right)$ is defined as a semi-simplicial complex and the reader is referred to [26] for information about semi-simplicial topology. A typical $n$-simplex of $\mathscr{W} h\left(\mathbf{R}^{m} \times F\right)$ consists of the equivalence class of a map $f: M \rightarrow \mathbf{R}^{m} \times F \times \Delta^{n}$, where there is a bundle $\rho: M \rightarrow \Delta^{n}$ with $Q$-manifold fiber, $M$ contains $\mathbf{R}^{m} \times F \times \Delta^{n}$ as a sliced $Z$-set, and $f$ is an f.p. $p^{-1}(\varepsilon)$-sdr for every $\varepsilon>0$. Another such map $f^{\prime}$ : $M^{\prime} \rightarrow \mathbf{R}^{m} \times F \times \Delta^{n}$ is equivalent to $f$ if there exists an f.p. homeomorphism $h$ : $M \rightarrow M^{\prime}$ such that $f^{\prime} h=f$ and $h \mid \mathbf{R}^{m} \times F \times \Delta^{n}=$ id.

The face and degeneracy operations in $\mathscr{W} h\left(\mathbf{R}^{m} \times F\right)$ are induced by the standard operations on $\Delta^{n}$. It follows immediately from the definitions that these operations are well defined.

The bundle $\rho: M \rightarrow \Delta^{n}$ and the sliced Z-embedding $\mathbf{R}^{m} \times F \times \Delta^{n} \rightarrow M$ will always be understood without further mention whenever a representative $f: M \rightarrow \mathbf{R}^{m}$ $\times F \times \Delta^{n}$ of an $n$-simplex of $\mathscr{W} h\left(\mathbf{R}^{m} \times F\right)$ is given.

The following lemma gives an alternative way of recognizing when a map represents an element of $\mathscr{W} h\left(\mathbf{R}^{m} \times F\right)$. First we need some more definitions. If $\alpha$ is an open cover of $Y$, then a proper map $f: X \rightarrow Y$ is said to be an $\alpha$-fibration if for all maps $F: Z \times[0,1] \rightarrow Y$ and $g: Z \rightarrow X$ for which $f g=F_{0}$, there is a map $G$ : $Z \times[0,1] \rightarrow X$ such that $G_{0}=g$ and $f G$ is $\alpha$-close to $F$. If $\varepsilon>0$, then we also use $\varepsilon$ to denote the open cover of $Y$ by balls of diameter $\varepsilon$. Thus, we speak of $\varepsilon$-fibrations. A map $f: X \rightarrow Y$ between ANRs is an approximate fibration provided it is an $\alpha$-fibration for every open cover $\alpha$ of $Y$. This notion was introduced in [14]. If $f$ : $X \times \Delta^{n} \rightarrow Y \times \Delta^{n}$ is an f.p. map, then $f$ is an approximate fibration if and only if $f_{t}$ : $X \times\{t\} \rightarrow Y \times\{t\}$ is an approximate fibration for each $t$ in $\Delta^{n}$. This follows from [15]. See [23] for the f.p. lifting property of an f.p. approximate fibration.

LEMMA 2.1. For every $\varepsilon>0$ there exists $a \delta=\delta(\varepsilon, m, n)>0$ such that if $\rho$ : $M \rightarrow \Delta^{n}$ is an ANR bundle, $M$ contains $\mathbf{R}^{m} \times F \times \Delta^{n}$, the inclusion $\mathbf{R}^{m} \times F \times \Delta^{n} \rightarrow M$ is f.p., f: $\boldsymbol{M} \rightarrow \mathbf{R}^{m} \times F \times \Delta^{n}$ is an f.p. sdr, and pf: $M \rightarrow \mathbf{R}^{m} \times \Delta^{n}$ is an f.p. $\delta$-fibration, then $f$ is an $f \cdot p \cdot p^{-1}(\varepsilon)$-sdr.

Conversely, if $f$ is an $f . p \cdot p^{-1}(\varepsilon)-s d r$, then $p f_{t}: M \rightarrow \mathbf{R}^{m}$ is an $\varepsilon$-fibration for each $t$ in $\Delta^{n}$.

Proof. If $p f$ were a Hurewicz fibration, then this would follow from Dold's proof that a fiber preserving homotopy equivalence between two Hurewicz fibrations is a fiber homotopy equivalence [16]. Just as in [8, Proposition 2.3] we note that Dold's proof can be adapted to the present situation by using only the approximate lifting property of $p f$. Note that $\delta$ depends on $m$ and $n$ as well as $\varepsilon$ because we need to deduce that $p f$ has an f.p. approximate lifting property (see [23, Theorem 2.4]).

The converse is easily verified.

The following corollary follows immediately from Lemma 2.1 and the definition of $\mathscr{W} h\left(\mathbf{R}^{m} \times F\right)$.

COROllaRy 2.2. If $\rho: M \rightarrow \Delta^{n}$ is a bundle with $Q$-manifold fiber and $M$ comtains $\mathbf{R}^{m} \times F \times \Delta^{n}$ as a sliced Z-set, then a map $f: M \rightarrow \mathbf{R}^{m} \times F \times \Delta^{n}$ represents an $n$-simplex of $\mathscr{W} h\left(\mathbf{R}^{m} \times F\right)$ if and only if $f$ is an $f . p$. sdr and $p f: M \rightarrow \mathbf{R}^{m} \times \Delta^{n}$ is an approximate fibration. 
The next theorem is an improvement of the main result of [24] in the special case when the base is euclidean space. This theorem describes an important property of parametrized families of approximate fibrations. This property can be summarized by saying that these families can be straightened out to be nearly like a product family.

TheOREM 2.3. Let $M$ be a $Q$-manifold, $q: M \times \Delta^{n} \times[0,1] \rightarrow \mathbf{R}^{m} \times \Delta^{n} \times[0,1]$ an approximate fibration which is f.p. over $\Delta^{n} \times[0,1]$, and let $\varepsilon>0$. Then there exists an f.p. homeomorphism $H: M \times \Delta^{n} \times[0,1] \rightarrow M \times \Delta^{n} \times[0,1]$ such that

(i) $H \mid M \times \Delta^{n} \times\{0\}=$ id;

(ii) $q H \mid M \times\{s\} \times\{t\}$ is $\varepsilon$-close to $q \mid M \times\{s\} \times\{0\}$ for each $(s, t)$ in $\Delta^{n} \times[0,1]$.

Moreover, there exists $a \delta=\delta(\varepsilon, m, n)>0$ such that if we are additionally given an $f$.p. homeom.oiphism $G: M \times \partial \Delta^{n} \times[0,1] \rightarrow M \times \partial \Delta^{n} \times[0,1]$ with the properties that $G \mid M \times \partial \Delta^{n} \times\{0\}=\mathrm{id}$ and that $g G \mid M \times\{s\} \times\{t\}$ is $\delta$-close to $q \mid M \times\{s\} \times\{0\}$ for each $(s, t)$ in $\partial \Delta^{n} \times[0,1]$, then we may additionally conclude that

(iii) $H \mid M \times \partial \Delta^{n} \times[0,1]=G$.

Furthermore, if we are additionally given that $M$ contains $\mathbf{R}^{m} \times F$ as a $Z$-set, $q$ : $\mathbf{R}^{m} \times F \times \Delta^{n} \times[0,1] \rightarrow \mathbf{R}^{m} \times \Delta^{n} \times[0,1]$ is projection, and $G \mid \mathbf{R}^{m} \times F \times \partial \Delta^{n} \times[0,1]$ $=\mathrm{id}$, then we may additionally conclude that

(iv) $H \mid \mathbf{R}^{m} \times F \times \Delta^{n} \times[0,1]=$ id.

Proof. In order to save space we assume that the reader is familiar with the proof of the main theorem in [24].

Let $g: M \times \Delta^{n} \rightarrow \mathbf{R}^{m} \times \Delta^{n}$ be defined by $g(x, s)=q(x, s, 0)$. Then $g \times \mathrm{id}_{[0,1]}$ is f.p. homotopic to $q$ rel $M \times \Delta^{n} \times\{0\}$. This homotopy can be approximately lifted in order to obtain an f.p. map $f: M \times \Delta^{n} \times[0,1] \rightarrow M \times \Delta^{n} \times[0,1]$ such that $f \mid M \times$ $\Delta^{n} \times\{0\}=$ id and $(g \times$ id) $f$ is close to $q$. Moreover, if we have the additional hypotheses of the theorem, then we can assume that $f \mid M \times \partial \Delta^{n} \times[0,1]=G^{-1}$ and that $f \mid \mathbf{R}^{m} \times F \times \Delta^{n} \times[0,1]=$ id.

Now the problem is to approximate $f$ by a homeomorphism. Specifically, we show that $f$ is f.p. homotopic $\operatorname{rel}\left(M \times \Delta^{n} \times\{0\}\right) \cup\left(M \times \partial \Delta^{n} \times[0,1]\right)$ to a homeomorphism $h: M \times \Delta^{n} \times[0,1] \rightarrow M \times \Delta^{n} \times[0,1]$ by a homotopy which is small when projected to $\mathbf{R}^{m} \times \Delta^{n} \times[0,1]$ by $g \times$ id. By sliced $Z$-set unknotting we may further assume that $h\left|\mathbf{R}^{m} \times F \times \Delta^{n} \times[0,1]=f\right|$. Then $H=h^{-1}$ will be the desired homeomorphism.

By following the proof of Theorem 3.1 in [24] we-obtain an f.p. map $\bar{h}$ : $M \times \Delta^{n} \times[0,1] \rightarrow M \times \Delta^{n} \times[0,1]$ such that $\bar{h}$ is a homeomorphism over

$$
g^{-1}\left(B_{3}^{m} \times \Delta^{n}\right) \times[0,1]
$$

and $f$ is f.p. homotopic to $\bar{h} \operatorname{rel}\left(M \times \Delta^{n} \times\{0\}\right) \cup\left(M \times \partial \Delta^{n} \times[0,1]\right)$ by a homotopy which is small when projected to $\mathbf{R}^{m} \times \Delta^{n} \times[0,1]$ by $g \times$ id. (We use $B_{r}^{m}$ to denote the $m$-cell $[-r, r]^{m}$ in $\mathbf{R}^{m}$.) It should be noted that the wrapping up construction in [24] is avoided here since the base is already $\mathbf{R}^{m}$. Thus we are only using that part of the proof of Theorem 3.1 in [24] which follows Assertion 3.2.

The final step is to use an infinite expanding trick to produce the homeomorphism $h$ promised above from the map $\bar{h}$. First, notice that by a small modification of $\bar{h}$ we 
can assume that there is a neighborhood $N$ of $\left(\Delta^{n} \times\{0\}\right) \cup\left(\partial \Delta^{n} \times[0,1]\right)$ in $\Delta^{n} \times$ $[0,1]$ such that $\bar{h}: M \times N \rightarrow M \times N$ is a homeomorphism.

Let $\Theta: \mathbf{R}^{m} \rightarrow \mathbf{R}^{m}$ be a radially defined homeomorphism such that $\Theta \mid B_{1}^{m}=\mathrm{id}$, $\Theta\left(B_{6}^{m}\right)=B_{2}^{m}$, and $\Theta$ is supported on $B_{7}^{m}$. Then $\Theta$ induces an f.p. homeomorphism $\bar{\Theta}: \mathbf{R}^{m} \times \Delta^{n} \times[0,1] \rightarrow \mathbf{R}^{m} \times \Delta^{n} \times[0,1]$ such that $\Theta \mid\left(\mathbf{R}^{m} \times \Delta^{n} \times\{0\}\right) \cup\left(\mathbf{R}^{m} \times \partial \Delta^{n}\right.$ $\times[0,1])=$ id and $\bar{\Theta}_{t}=\Theta$ for $t \in\left(\Delta^{n} \times[0,1]\right) \backslash N$. By the parametrized engulfing theorem in [23, Theorem 4.3] there are f.p. homeomorphisms $\tilde{\Theta}^{i}: M \times \Delta^{n} \times[0,1] \rightarrow$ $M \times \Delta^{n} \times[0,1]$ for $i=1,2$ such that $\tilde{\Theta}^{i} \mid\left(M \times \Delta^{n} \times\{0\}\right) \cup\left(M \times \partial \Delta^{n} \times[0,1]\right)=\mathrm{id}$, $q \tilde{\Theta}^{1}$ is close to $\bar{\Theta} q,(g \times$ id $) \tilde{\Theta}^{2}$ is close to $\bar{\Theta}(g \times$ id $)$,

$$
\tilde{\Theta}^{1}=\text { id on } q^{-1}\left(B_{1}^{m} \times \Delta^{n} \times[0,1]\right),
$$

and

$$
\tilde{\Theta}^{2}=\text { id on } g^{-1}\left(B_{1}^{m} \times \Delta^{n}\right) \times[0,1]
$$

Then

$$
\bar{h}_{1}=\left(\tilde{\Theta}^{2}\right)^{-1} \bar{h} \tilde{\Theta}^{1}: M \times \Delta^{n} \times[0,1] \rightarrow M \times \Delta^{n} \times[0,1]
$$

is a homeomorphism over $g^{-1}\left(B_{6}^{m} \times \Delta^{n}\right) \times[0,1]$. Also there is a small (measured in $\left.\mathbf{R}^{m} \times \Delta^{n} \times[0,1]\right)$ f.p. homotopy from $\bar{h}_{1}$ to $\bar{h}$ which comes from using isotopies $\mathrm{id} \simeq \tilde{\Theta}^{i}$ for $i=1,2$ provided by [23, Theorem 4.3]. It is clear that one can continue this process to obtain a sequence of maps $\bar{h}_{1}, \bar{h}_{2}, \ldots$ such that $h=\lim _{i \rightarrow \infty} \bar{h}_{i}$ is the required homeomorphism. The reader should consult [8, pp. 327, 328] for a similar construction.

3. Homotopy and torsion in $\mathscr{W} h\left(\mathbf{R}^{m} \times F\right)$. This section begins by showing how to deform certain controlled homotopy equivalences to maps which represent simplices of $\mathscr{W} h\left(\mathbf{R}^{m} \times F\right)$. This is contained in Propositions 3.1 and 3.2. Next, Proposition 3.4 gives a geometric way to understand what it means for two $n$-simplices of

$$
\mathscr{W} h\left(\mathbf{R}^{m} \times F\right)
$$

to represent the same homotopy class in $\pi_{n} \mathscr{W} h\left(\mathbf{R}^{m} \times F\right)$. Finally, we define the torsion of certain controlled homotopy equivalences to be an element of

$$
\pi_{*} \mathscr{W} h\left(\mathbf{R}^{m} \times F\right)
$$

and characterize geometrically what it means for two of these homotopy equivalences to have the same torsion (Proposition 3.6).

We begin with some more definitions. If $\alpha$ is an open cover of $Y$, then a proper map $f: X \rightarrow Y$ is an $\alpha$-equivalence provided there is a proper map $g: Y \rightarrow X$ and proper homotopies $g f \simeq$ id and $f g \simeq$ id limited by $f^{-1}(\alpha)$ and $\alpha$, respectively. If $X$ and $Y$ are both fibered over a space, then $f$ is an f.p. $\alpha$-equivalence provided $f, g$ and the homotopies are all f.p. If $Y=\mathbf{R}^{m} \times F$, then $f: X \rightarrow \mathbf{R}^{m} \times F$ is a bounded homotopy equivalence if it is a $p^{-1}(\varepsilon)$-equivalence for some (possibly large) $\varepsilon>0$.

Proposition 3.1. For every $\varepsilon>0$ there exists $a \delta=\delta(\varepsilon, m, n)>0$ such that if $\rho$ : $M \rightarrow \Delta^{n}$ is a bundle with $Q$-manifold fiber and $f: M \rightarrow \mathbf{R}^{m} \times F \times \Delta^{n}$ is an f.p. $p^{-1}(\delta)$-equivalence, then $f$ is $f . p \cdot p^{-1}(\varepsilon)$-homotopic to a map $\tilde{f}: M \rightarrow \mathbf{R}^{m} \times F \times \Delta^{n}$ which is an $f . p \cdot p^{-1}(\mu)$-equivalence for every $\mu>0$. 
Moreover, if it is additionally given that $M$ contains $\mathbf{R}^{m} \times F \times \Delta^{n}$ as a sliced Z-set and $f \mid \mathbf{R}^{m} \times F \times \Delta^{n}=\mathrm{id}$, then we may additionally conclude that the homotopy from $f$ to $\tilde{f}$ is rel $\mathbf{R}^{m} \times F \times \Delta^{n}$.

Furthermore, if it is additionally given that $f \mid: \rho^{-1}\left(\partial \Delta^{n}\right) \rightarrow \mathbf{R}^{m} \times F \times \partial \Delta^{n}$ is an f.p. $p^{-1}(\mu)$-equivalence for every $\mu>0$, then we may additionally conclude that the homotopy from $f$ to $\tilde{f}$ is $\operatorname{rel} \rho^{-1}\left(\partial \Delta^{n}\right)$.

Proof. The f.p. map $p f: M \rightarrow \mathbf{R}^{m} \times \Delta^{n}$ has the property that $p f_{t}: \rho^{-1}(t) \rightarrow \mathbf{R}^{m}$ is a $\delta$-fibration for each $t$ in $\Delta^{n}$. If $\delta$ is small enough, then it follows from Theorem 7.2 and Remark 7.5 of [23] that $p f$ is f.p. $\varepsilon$-homotopic to a map $g: M \rightarrow \mathbf{R}^{m} \times \Delta^{n}$ which is an f.p. approximate fibration. Lift this homotopy to get an f.p. $p^{-1}(\varepsilon)$-homotopy from $f$ to a map $\tilde{f}$ such that $p \tilde{f}=g$. Since $\tilde{f}$ is an f.p. homotopy equivalence and $p \tilde{f}$ is an f.p. approximate fibration, the method of Dold used in the proof of Lemma 2.1 shows that $\tilde{f}$ is an f.p. $p^{-1}(\mu)$-equivalence for every $\mu>0$.

If we have the additional hypotheses of the proposition, then one uses the full strength of Theorem 7.2 of [23] to make the homotopy from $p f$ to $g \operatorname{rel}\left(\mathbf{R}^{m} \times F \times \Delta^{n}\right)$ $\cup \rho^{-1}\left(\partial \Delta^{n}\right)$. Then the lifted homotopy from $f$ to $\tilde{f}$ can be required to have this same property.

The next proposition is the analogue of Proposition 3.1 for bounded equivalences.

Proposition 3.2. If $\rho: M \rightarrow \Delta^{n}$ is a bundle with $Q$-manifold fiber and $f$ : $M \rightarrow \mathbf{R}^{m} \times F \times \Delta^{n}$ is an $f$.p. bounded equivalence, then $f$ is $f$. p. boundedly homotopic to a map $\tilde{f}: M \rightarrow \mathbf{R}^{m} \times F \times \Delta^{n}$ which is an $f . p \cdot p^{-1}(\mu)$-equivalence for every $\mu>0$.

Moreover, the last two paragraphs of Proposition 3.1 hold verbatim in this situation.

Proof. There exists a $c>0$ such that $p f_{t}: \rho^{-1}(t) \rightarrow \mathbf{R}^{m}$ is a $c$-fibration for each $t$ in $\Delta^{n}$. Choose $K>0$ large and let $\gamma: \mathbf{R}^{m} \rightarrow \mathbf{R}^{m}$ be the homeomorphism defined by $\gamma(x)=x / K$. Then each $\gamma p f_{t}: \rho^{-1}(t) \rightarrow \mathbf{R}^{m}$ is a $\delta$-fibration, where $\delta>0$ is small. As in the proof of Proposition 3.1 we can find a small f.p. homotopy of $(\gamma \times$ id) $p f$ to an f.p. approximate fibration $g: M \rightarrow \mathbf{R}^{m} \times \Delta^{n}$. Then $\left(\gamma^{-1} \times\right.$ id $) g$ is still an f.p. approximate fibration and it is f.p. boundedly homotopic to $p f$. Now lift this bounded homotopy as in the proof of Proposition 3.1 in order to obtain the desired $\operatorname{map} \tilde{f}$.

If the additional hypotheses of the proposition are given, then one only has to replace the homeomorphism $\gamma \times$ id: $\mathbf{R}^{m} \times \Delta^{n} \rightarrow \mathbf{R}^{m} \times \Delta^{n}$ by an f.p. homeomorphism $\bar{\gamma}$ which has the property that $\bar{\gamma} \mid \mathbf{R}^{m} \times \partial \Delta^{n}=\mathrm{id}$.

Since $\mathscr{W} h\left(\mathbf{R}^{m} \times F\right)$ satisfies the Kan extension condition, it makes sense to talk about the homotopy groups of $\mathscr{W} h\left(\mathbf{R}^{m} \times F\right)$. The $n$th homotopy group is based at the $n$-simplex of $\mathscr{W} h\left(\mathbf{R}^{m} \times F\right)$ which is represented by the projection map $\mathbf{R}^{m} \times F$ $\times[0,1] \times \Delta^{n} \rightarrow \mathbf{R}^{m} \times F \times \Delta^{n}$, where the sliced Z-embedding of $\mathbf{R}^{m} \times F \times \Delta^{n}$ into $\mathbf{R}^{m} \times F \times[0,1] \times \Delta^{n}$ is given by inclusion into the 0-level. If $f: M \rightarrow \mathbf{R}^{m} \times F \times \Delta^{n}$ represents an $h$-simplex of $\mathscr{W} h\left(\mathbf{R}^{m} \times F\right)$ which determines a class in $\pi_{n} \mathscr{W} h\left(\mathbf{R}^{m} \times F\right)$, then that class is denoted by $[f]$. Such a map has the property that $f \mid: \rho^{-1}\left(\partial_{i} \Delta^{n}\right) \rightarrow$ $\mathbf{R}^{m} \times F \times \partial_{i} \Delta^{n}$ is equivalent to the "base $(n-1)$-simplex" of $\mathscr{W} h\left(\mathbf{R}^{m} \times F\right)$ for each $i=0,1, \ldots, n$. The following lemma will be useful in analyzing these maps. 
LEMMA 3.3. Suppose that $f: M \rightarrow \mathbf{R}^{m} \times F \times \Delta^{n}$ represents a class [ $\left.f\right]$ in

$$
\pi_{n} \mathscr{W} h\left(\mathbf{R}^{m} \times F\right) \text {. }
$$

Then there exists an f.p. homeomorphism $j: \mathbf{R}^{m} \times F \times[0,1] \times \partial \Delta^{n} \rightarrow \rho^{-1}\left(\partial \Delta^{n}\right)$ such that $j \mid \mathbf{R}^{m} \times F \times\{0\} \times \partial \Delta^{n}=$ id and $f j: \mathbf{R}^{m} \times F \times[0,1] \times \partial \Delta^{n} \rightarrow \mathbf{R}^{m} \times F \times \partial \Delta^{n}$ is projection.

Proof. Since $f$ represents a class of $\pi_{n} \mathscr{W} h\left(\mathbf{R}^{m} \times F\right)$, it follows that there are f.p. homeomorphisms $g_{i}: \mathbf{R}^{m} \times F \times[0,1] \times \partial_{i} \Delta^{n} \rightarrow \rho^{-1}\left(\partial_{i} \Delta^{n}\right)$ for $i=0,1, \ldots, n$ such that $g_{i} \mid \mathbf{R}^{m} \times F \times\{0\} \times \partial_{i} \Delta^{n}=\mathrm{id}$ and $f g_{i}$ is projection. For each $i=1,2, \ldots, n$ let $C_{i}$ denote the subset of the boundary of $\partial_{i} \Delta^{n}$ which meets $\bigcup_{k=0}^{i-1} \partial_{k} \Delta^{n}$. The $g_{i}$ 's will now be modified so that they agree on their common domain.

We will inductively define f.p. homeomorphisms $g_{0}=\bar{g}_{0}, \bar{g}_{1}, \ldots, \bar{g}_{n}=j$, where

$$
\bar{g}_{i}: \mathbf{R}^{m} \times F \times[0,1] \times\left(\bigcup_{k=0}^{i} \partial_{k} \Delta^{n}\right) \rightarrow \rho^{-1}\left(\bigcup_{k=0}^{i} \partial_{k} \Delta^{n}\right) .
$$

These are defined so that $\bar{g}_{i+1}$ extends $\bar{g}_{i}, \bar{g}_{i} \mid \mathbf{R}^{m} \times F \times\{0\} \times\left(\cup_{k=0}^{i} \partial_{k} \Delta^{n}\right)=\mathrm{id}$, and $f \bar{g}_{i}$ is projection. Assuming $i \geqslant 1$ and that $\bar{g}_{i-1}$ has been defined, proceed to define $\bar{g}_{i}$ on $\mathbf{R}^{m} \times F \times[0,1] \times \partial_{i} \Delta^{n}$. To this end consider

$$
g_{i}^{-1} \bar{g}_{i-1} \mid: \mathbf{R}^{m} \times F \times[0,1] \times C_{i} \rightarrow \mathbf{R}^{m} \times F \times[0,1] \times C_{i} .
$$

This is a homeomorphism whose restriction to $\mathbf{R}^{m} \times F \times\{0\} \times C_{i}$ is the identity. In addition, this homeomorphism affects only the $[0,1]$-coordinate of any point. It follows that $g_{i}^{-1} \bar{g}_{i-1} \mid$ extends to a homeomorphism

$$
g: \mathbf{R}^{m} \times F \times[0,1] \times \partial_{i} \Delta^{n} \rightarrow \mathbf{R}^{m} \times F \times[0,1] \times \partial_{i} \Delta^{n}
$$

such that $g \mid \mathbf{R}^{m} \times F \times\{0\} \times \partial_{i} \Delta^{n}=$ id and $g$ affects only the [0,1]-coordinate of any point. Define $\bar{g}_{i} \mid \mathbf{R}^{m} \times F \times[0,1] \times \partial_{i} \Delta^{n}$ to be $g_{i} g$.

The next result shows how to detect the equivalence of elements in $\pi_{\eta} \mathscr{W} h\left(\mathbf{R}^{m} \times F\right)$. Two maps $f, g: M \rightarrow \mathbf{R}^{m} \times F \times \Delta^{n}$ are said to be boundedly close if there exists a constant $L>0$ such that $p f$ is $L$-close to $p g$, where $p$ denotes projection to $\mathbf{R}^{m}$.

Proposition 3.4. Let $f: M \rightarrow \mathbf{R}^{m} \times F \times \Delta^{n}$ and $f^{\prime}: M^{\prime} \rightarrow \mathbf{R}^{m} \times F \times \Delta^{n}$ represent the elements $[f]$ and $\left[f^{\prime}\right]$ of $\pi_{n} \mathscr{W} h\left(\mathbf{R}^{m} \times F\right)$, respectively. Then the following are equivalent:

(i) $[f]=\left[f^{\prime}\right]$;

(ii) for every $\varepsilon>0$ there exists an $f . p$. homeomorphism $h: M \rightarrow M^{\prime}$ such that $h\left|\mathbf{R}^{m} \times F \times \Delta^{n}=\mathrm{id}, f^{\prime} h\right| \rho^{-1}\left(\partial \Delta^{n}\right)=f \mid \rho^{-1}\left(\partial \Delta^{n}\right)$, and $f^{\prime} h$ is $f . p \cdot p^{-1}(\varepsilon)$-homotopic to $f$ $\operatorname{rel}\left(\mathbf{R}^{m} \times F \times \Delta^{n}\right) \cup \rho^{-1}\left(\partial \Delta^{n}\right)$;

(iii) there exists on f.p. homeomorphism $h: M \rightarrow M^{\prime}$ such that $h \mid \mathbf{R}^{m} \times F \times \Delta^{n}=\mathrm{id}$, $f^{\prime} h\left|\rho^{-1}\left(\partial \Delta^{n}\right)=f\right| \rho^{-1}\left(\partial \Delta^{n}\right)$, and $f^{\prime} h$ is boundedly close to $f$.

Proof. It is first shown that condition (i) implies condition (ii). Since $[f]=\left[f^{\prime}\right]$ there is a bundle $\tilde{\rho}: \tilde{M} \rightarrow \Delta^{n} \times I$ with $Q$-manifold fiber and an f.p. (over $\Delta^{n} \times I$ ) map $\tilde{f}: \tilde{M} \rightarrow \mathbf{R}^{m} \times F \times \Delta^{n} \times I$ representing an $(n+1)$-simplex of $\mathscr{W} h\left(\mathbf{R}^{m} \times F\right)$ such that $\tilde{f} \mid \tilde{\rho}^{-1}\left(\Delta^{n} \times\{0\}\right)$ represents $[f], \tilde{f} \mid \tilde{\rho}^{-1}\left(\Delta^{n} \times\{1\}\right)$ represents $\left[f^{\prime}\right]$, and $\tilde{f} \mid \tilde{\rho}^{-1}\left(\partial_{i} \Delta^{n} \times I\right)$ represents the "base $n$-simplex" of $\mathscr{W} h\left(\mathbf{R}^{m} \times F\right)$ for all $i$. 
By (the proof of) Lemma 3.3 there is f.p. homeomorphism $j: \mathbf{R}^{m} \times F \times[0,1] \times$ $\partial \Delta^{n} \times I \rightarrow \tilde{\rho}^{-1}\left(\partial \Delta^{n} \times I\right)$ such that $j \mid \mathbf{R}^{m} \times F \times\{0\} \times \partial \Delta^{n} \times I=$ id and $\tilde{f j}$ is projection.

Using the fact that $\tilde{\rho}$ is trivial and sliced $Z$-set unknotting, one can construct an f.p. homeomorphism $\tilde{h}: \mathbf{R}^{m} \times F \times[0,1] \times \Delta^{n} \times I \rightarrow \tilde{M}$ such that $\tilde{h} \mid \mathbf{R}^{m} \times F \times\{0\}$ $\times \Delta^{n} \times I=$ id (see Lemma 5.1).

Let $j_{0}=j \mid \mathbf{R}^{m} \times F \times[0,1] \times \Delta^{n} \times\{0\}$ and let $\tilde{h}_{0}=\tilde{h} \mid \mathbf{R}^{m} \times F \times[0,1] \times \Delta^{n} \times\{0\}$. By Theorem 2.3 there is an f.p. homeomorphism

$$
H: \mathbf{R}^{m} \times F \times[0,1] \times \Delta^{n} \times I \rightarrow \mathbf{R}^{m} \times F \times[0,1] \times \Delta^{n} \times I
$$

with the following properties:

(1) $H \mid \mathbf{R}^{m} \times F \times[0,1] \times \Delta^{n} \times\{0\}=$ id;

(2) $H\left|\mathbf{R}^{m} \times F \times[0,1] \times \partial \Delta^{n} \times I=\tilde{h}^{-1} \circ j \circ\left(j_{0}^{-1} \times \mathrm{id}_{I}\right) \circ\left(\tilde{h}_{0} \times \mathrm{id}_{I}\right)\right|$;

(3) $H \mid \mathbf{R}^{m} \times F \times\{0\} \times \Delta^{n} \times I=$ id;

(4) $p \tilde{f} \tilde{h} H \mid \mathbf{R}^{m} \times F \times[0,1] \times\{s\} \times\{t\}$ is $\varepsilon$-close to $p \tilde{f} \tilde{h} \mid \mathbf{R}^{m} \times F \times[0,1] \times\{s\} \times$ $\{0\}$ for each $(s, t)$ in $\Delta^{n} \times I$.

Since $\tilde{f} \mid \tilde{\rho}^{-1}\left(\Delta^{n} \times\{0\}\right)$ represents $[f]$, there is an f.p. homeomorphism $\alpha: M \rightarrow$ $\tilde{\rho}^{-1}\left(\Delta^{n} \times\{0\}\right)$ such that $\tilde{f} \boldsymbol{\alpha}=f$ and $\alpha \mid \mathbf{R}^{m} \times F \times \Delta^{n}=$ id. Likewise, there is an f.p. homeomorphism $\beta: M^{\prime} \rightarrow \tilde{\rho}^{-1}\left(\Delta^{n} \times\{1\}\right)$ such that $\tilde{f} \beta=f^{\prime}$ and $\beta \mid \mathbf{R}^{m} \times F \times \Delta^{n}=$ id.

Now the required homeomorphism $h: M \rightarrow M^{\prime}$ can be described by the composition

$$
\begin{aligned}
M & \stackrel{\alpha}{\rightarrow} \tilde{\rho}^{-1}\left(\Delta^{n} \times\{0\}\right) \stackrel{\tilde{h}_{0}^{-1}}{\rightarrow} \mathbf{R}^{m} \times F \times[0,1] \times \Delta^{n} \times\{0\} \\
& \stackrel{\text { id }}{\rightarrow} \mathbf{R}^{m} \times F \times[0,1] \times \Delta^{n} \times\{1\} \stackrel{H \mid}{\rightarrow} \mathbf{R}^{m} \times F \times[0,1] \times \Delta^{n} \times\{1\} \\
& \stackrel{\tilde{h}}{\rightarrow} \tilde{\rho}^{-1}\left(\Delta^{n} \times\{1\}\right) \stackrel{\beta^{-1}}{\rightarrow} M^{\prime} .
\end{aligned}
$$

The homotopy from $f$ to $f^{\prime} h$ is given at time $t$ by the composition

$$
\begin{aligned}
M & \stackrel{\alpha}{\rightarrow} \tilde{\rho}^{-1}\left(\Delta^{n} \times\{0\}\right) \stackrel{\tilde{h}_{0}^{-1}}{\rightarrow} \mathbf{R}^{m} \times F \times[0,1] \times \Delta^{n} \times\{0\} \\
& \stackrel{\text { id }}{\rightarrow} \mathbf{R}^{m} \times F \times[0,1] \times \Delta^{n} \times\{t\} \stackrel{H \mid}{\rightarrow} \mathbf{R}^{m} \times F \times[0,1] \times \Delta^{n} \times\{t\} \\
& \stackrel{\tilde{h} \mid}{\rightarrow} \tilde{\rho}^{-1}\left(\Delta^{n} \times\{t\}\right) \stackrel{\tilde{f} \mid}{\rightarrow} \mathbf{R}^{m} \times F \times \Delta^{n} \times\{t\}=\mathbf{R}^{m} \times F \times \Delta^{n} .
\end{aligned}
$$

This proves that (i) implies (ii).

Since (ii) obviously implies (iii), it remains to show that (iii) implies (i). The following assertion first shows how to replace $f$ by a map which has a particularly standard form over $\partial \Delta^{n}$.

AsSERTION 3.4.1. $f$ is f.p. boundedly homotopic $\operatorname{rel}\left(\mathbf{R}^{m} \times F \times \Delta^{n}\right) \cup \rho^{-1}\left(\partial \Delta^{n}\right)$ to a map $\tilde{f}$ which has the property that there is an f.p. homotopy $G: \operatorname{id}_{M} \simeq \tilde{f} \operatorname{rel}^{m} \times F$ $\times \Delta^{n}$ such that $\tilde{f} G$ is a bounded homotopy and $\tilde{f} G \mid \rho^{-1}\left(\partial \Delta^{n}\right)$ is a stationary homotopy.

Proof. By Lemma 3.3 there is an f.p. homeomorphism $j: \mathbf{R}^{m} \times F \times[0,1] \times \partial \Delta^{n}$ $\rightarrow \rho^{-1}\left(\partial \Delta^{n}\right)$ such that $j \mid \mathbf{R}^{m} \times F \times\{0\} \times \partial \Delta^{n}=$ id and $f j$ is projection. Since $\rho$ is trivial there is an f.p. homeomorphism $\tilde{h}: \mathbf{R}^{m} \times F \times[0,1] \times \Delta^{n} \rightarrow M$. By sliced $Z$-set 
unknotting it is also possible to assume that $\tilde{h} \mid \mathbf{R}^{m} \times F \times\{0\} \times \Delta^{n}=$ id and $\tilde{h} \mid \mathbf{R}^{m} \times$ $F \times[0,1] \times \partial_{n} \Delta^{n}=j \mid$ (see Lemma 5.1). By Theorem 2.3 there is an f.p. homeomorphism $H: \mathbf{R}^{m} \times F \times[0,1] \times \Delta^{n} \rightarrow \mathbf{R}^{m} \times F \times[0,1] \times \Delta^{n}$ such that $H \mid \mathbf{R}^{m} \times F \times\{0\}$ $\times \Delta^{n}=\mathrm{id}, H \mid \mathbf{R}^{m} \times F \times[0,1] \times \partial_{n} \Delta^{n}=\mathrm{id}$, and $p \tilde{f} H: \mathbf{R}^{m} \times F \times[0,1] \times \Delta^{n} \rightarrow \mathbf{R}^{m}$ $\times \Delta^{n}$ is close to projection.

We want to extend $j$ over a neighborhood of $\partial \Delta^{n}$ as follows. Let $\partial \Delta^{n} \times[0,1]$ be a closed collar of $\partial \Delta^{n}$ in $\Delta^{n}$ so that $\partial \Delta^{n} \times\{0\}$ is identified with $\partial \Delta^{n}$. Define $\tilde{j}$ : $\mathbf{R}^{m} \times F \times[0,1] \times \partial \Delta^{n} \times[0,1] \rightarrow \rho^{-1}\left(\partial \Delta^{n} \times[0,1]\right)$ by $\tilde{j}=\tilde{h} H\left[\left(H^{-1} \tilde{h}^{-1} j\right) \times \mathrm{id}_{[0,1]}\right]$. Note that $\tilde{j}$ extends $j$, that $\tilde{j} \mid \mathbf{R}^{m} \times F \times\{0\} \times \partial \Delta^{n} \times[0,1]=\mathrm{id}$, and that $p f \tilde{j}$ is close to $\operatorname{proj}\left[H^{-1} \tilde{h}^{-1} j \times \mathrm{id}\right]$.

Now define an f.p. homotopy $\hat{G}$ on $M$ by defining $\hat{G}_{\alpha}: M \rightarrow M, 0 \leqslant \alpha \leqslant 1$, as follows. First

$$
\hat{G}_{\alpha} \mid \rho^{-1}\left(\Delta^{n} \backslash\left(\partial \Delta^{n} \times[0,1]\right)\right)=\text { id } \text { for } 0 \leqslant \alpha \leqslant 1 .
$$

Then for $(x, f, s, t, u)$ in $\mathbf{R}^{m} \times F \times[0,1] \times \partial \Delta^{n} \times[0,1]$ set $\hat{G}_{\alpha} \tilde{j}(x, f, s, t, u)=$ $j(x, f,(1-\alpha) s+\alpha u s, t, u)$. Note that $f \hat{G}$ is a bounded homotopy and that $f \hat{G}$ is $\operatorname{rel}\left(\mathbf{R}^{m} \times F \times \Delta^{n}\right) \cup \rho^{-1}\left(\partial \Delta^{n}\right)$.

Define $\tilde{f}: M \rightarrow \mathbf{R}^{m} \times F \times \Delta^{n}$ by setting $\tilde{f}=f \hat{G}_{1}$. It remains to show that $G$ exists. To this end let $K$ : id ${ }_{M} \simeq f$ be an f.p. homotopy rel $\mathbf{R}^{m} \times F \times \Delta^{n}$ such that $f K$ is a bounded homotopy. Define $G$ by setting

$$
G_{\alpha}= \begin{cases}\hat{G}_{2 \alpha} & \text { for } 0 \leqslant \alpha \leqslant \frac{1}{2}, \\ K_{2 \alpha-1} \circ \hat{G}_{1} & \text { for } \frac{1}{2} \leqslant \alpha \leqslant 1\end{cases}
$$

This completes the proof of Assertion 3.4.1.

The following assertion shows the existence of a homotopy from $f$ to $f^{\prime} h$.

ASSERTION 3.4.2. $f$ is f.p. boundedly homotopic to $f^{\prime} h \operatorname{rel}\left(\mathbf{R}^{m} \times F \times \Delta^{n}\right) \cup$ $\rho^{-1}\left(\partial \Delta^{n}\right)$.

Proof. Let $f \hat{G}: f \simeq \tilde{f}$ and $G: \mathrm{id}_{M} \simeq \tilde{f}$ be the homotopies defined in the proof of Assertion 3.4.1. Then the desired homotopy $L: f \simeq f^{\prime} h$ is defined by setting

$$
L_{\alpha}= \begin{cases}f \hat{G}_{2 \alpha} & \text { for } 0 \leqslant \alpha \leqslant \frac{1}{2} \\ f^{\prime} h G_{2-2 \alpha} & \text { for } \frac{1}{2} \leqslant \alpha \leqslant 1\end{cases}
$$

To complete the proof of the proposition, define $\hat{L}: M \times I \rightarrow \mathbf{R}^{m} \times F \times \Delta^{n} \times I$ by setting $\hat{L}(x, \alpha)=\left(L_{\alpha}(x), \alpha\right)$ for $(x, \alpha)$ in $M \times I$, where $L_{\alpha}$ is defined in the proof of Assertion 3.4.2. Then $\hat{L}$ is an f.p. bounded sdr. And by Proposition 3.2, $\hat{L}$ is f.p. boundedly homotopic $\operatorname{rel}\left(\mathbf{R}^{m} \times F \times \Delta^{n} \times I\right) \cup\left(\rho^{-1}\left(\partial \Delta^{n}\right) \times I\right)$ to $\tilde{L}$ which is an f.p. $p^{-1}(\mu)$-equivalence for every $\mu>0$. This $\tilde{L}$ defines an $(n+1)$-simplex in

$$
\mathscr{W} h\left(\mathbf{R}^{m} \times F\right)
$$

showing that $[f]=\left[f^{\prime} h\right]$ in $\pi_{n} \mathscr{W} h\left(\mathbf{R}^{m} \times F\right)$. Since $f^{\prime}$ is equivalent to $f^{\prime} h$ in $\mathscr{W} h\left(\mathbf{R}^{m} \times F\right)$, this completes the proof.

The remainder of this section is devoted to defining and studying the torsion of certain homotopy equivalences to $\mathbf{R}^{m} \times F \times \Delta^{n}$. 
Consider the following data:

(I) a bundle $\rho: M \rightarrow \Delta^{n}$ with $Q$-manifold fiber;

(II) a sliced $Z$-embedding $\mathbf{R}^{m} \times F \times \partial \Delta^{n} \subset \rho^{-1}\left(\partial \Delta^{n}\right)$;

(III) an f.p. bounded homotopy equivalence $f: M \rightarrow \mathbf{R}^{m} \times F \times \Delta^{n}$ such that each $(n-1)$-face of $f, f \mid: \rho^{-1}\left(\partial_{i} \Delta^{n}\right) \rightarrow \mathbf{R}^{m} \times F \times \partial_{i} \Delta^{n}$ for $i=0,1, \ldots, n$, is equivalent to the "base $(n-1)$-simplex" of $\mathscr{W} h\left(\mathbf{R}^{m} \times F\right)$.

When this data is given we will define the torsion of $f$. The immediate goal is to find an f.p. bounded homotopy rel $\rho^{-1}\left(\partial \Delta^{n}\right)$ of $f$ to a map which represents a class in $\pi_{n} \mathscr{W} h\left(\mathbf{R}^{m} \times F\right)$. To this end let $g: \mathbf{R}^{m} \times F \times \Delta^{n} \rightarrow M$ be an f.p. bounded homotopy inverse for $f$. We may assume that $g$ is a sliced $Z$-embedding and that $g \mid \mathbf{R}^{m} \times F \times$ $\partial \Delta^{n}=$ id by sliced $Z$-set unknotting. Identify $\mathbf{R}^{m} \times F \times \Delta^{n}$ with its image under $g$ and regard $g$ as an inclusion map.

Now $f$ is f.p. boundedly homotopic rel $\rho^{-1}\left(\partial \Delta^{n}\right)$ to a map $\hat{f}: M \rightarrow \mathbf{R}^{m} \times F \times \Delta^{n}$ which is an f.p. bounded strong deformation retraction. This follows from the usual method of turning a weak deformation retraction into a strong deformation retraction (see [32, p. 31]).

By Proposition 3.2, $\hat{f}$ is f.p. boundedly homotopic $\operatorname{rel}\left(\mathbf{R}^{m} \times F \times \Delta^{n}\right) \cup \rho^{-1}\left(\partial \Delta^{n}\right)$ to a map $\tilde{f}: M \rightarrow \mathbf{R}^{m} \times F \times \Delta^{n}$ which is an f.p. $p^{-1}(\varepsilon)$-sdr for every $\varepsilon>0$. Then $\tilde{f}$ represents a class $[\tilde{f}]$ in $\pi_{n} \mathscr{W} h\left(\mathbf{R}^{m} \times F\right)$ and we define the torsion $\tau(f)$ of $f$ by $\tau(f)=[\tilde{f}]$.

Proposition 3.5. $\tau(f)$ is well defined.

Proof. Suppose $g^{\prime}: \mathbf{R}^{m} \times F \times \Delta^{n} \rightarrow M$ is another f.p. bounded homotopy inverse for $f$ such that $g^{\prime}$ is a sliced Z-embedding and $g^{\prime} \mid \mathbf{R} \times F \times \partial \Delta=\mathrm{id}$. Then $g^{\prime}$ gives rise to another class $\left[\tilde{f}^{\prime}\right]$ in $\pi_{n} \mathscr{W} h\left(\mathbf{R}^{m} \times F\right)$. Here $\tilde{f}^{\prime}$ is f.p. boundedly homotopic $\operatorname{rel} \rho^{-1}\left(\partial \Delta^{n}\right)$ to $f$. We must show $[\tilde{f}]=\left[\tilde{f}^{\prime}\right]$.

Note that $g$ is f.p. homotopic to $g^{\prime}$ by a homotopy which is bounded when projected to $\mathbf{R}^{m} \times F \times \Delta^{n}$ by $f$. By sliced $Z$-set unknotting there exists an f.p. homeomorphism $h: M \rightarrow M$ such that $h g=g^{\prime}$ and $f h$ is boundedly close to $f$. Since the homotopy from $g$ to $g^{\prime}$ can be chosen to be rel $\mathbf{R}^{m} \times F \times \partial \Delta^{n}$, it can be assumed that $h \mid \rho^{-1}\left(\partial \Delta^{n}\right)=\mathrm{id}$. That $[\tilde{f}]=\left[\tilde{f}^{\prime}\right]$ now follows from Proposition 3.4.

Observe that if $[f]$ is in $\pi_{n} \mathscr{W} h\left(\mathbf{R}^{m} \times F\right)$, then $\pi(f)=[f]$. The next proposition shows how to decide if two torsions are equal.

Proposition 3.6. Let $f: M \rightarrow \mathbf{R}^{m} \times F \times \Delta^{n}$ and $f^{\prime}: M^{\prime} \rightarrow \mathbf{R}^{m} \times F \times \Delta^{n}$ be maps for which $\tau(f)$ and $\tau\left(f^{\prime}\right)$ are defined. Then $\tau(f)=\tau\left(f^{\prime}\right)$ if and only if there is an $f . p$. homeomorphism $h: M \rightarrow M^{\prime}$ such that $h\left|\mathbf{R}^{m} \times F \times \partial \Delta^{n}=\mathrm{id}, f^{\prime} h\right| \rho^{-1}\left(\partial \Delta^{n}\right)=$ $f \mid \rho^{-1}\left(\partial \Delta^{n}\right)$, and $f^{\prime} h$ is $f$. p. boundedly homotopic rel $\rho^{-1}\left(\partial \Delta^{n}\right)$ to $f$.

Proof. If $\tau(f)=\tau\left(f^{\prime}\right)$, then the definition of torsion and Proposition 3.4 immediately imply the existence of $h$.

On the other hand, suppose the homeomorphism $h$ is given. To define the torsion $\tau(f)$ choose a sliced Z-embedding $g: \mathbf{R}^{m} \times F \times \Delta^{n} \rightarrow M$ such that $g$ is an f.p. bounded homotopy inverse for $f$ and $g \mid \mathbf{R}^{m} \times F \times \partial \Delta^{n}=$ id. It follows that $h g$ : $\mathbf{R}^{m} \times F \times \Delta^{n} \rightarrow M^{\prime}$ is an f.p. bounded homotopy inverse for $f^{\prime}$. Now Proposition 3.4 can be used to conclude that $\tau(f)=\tau\left(f^{\prime}\right)$. 
The next proposition is a version of Proposition 3.6 when the bounded homotopy equivalences have small $\delta$ control.

Proposition 3.7. For every $\varepsilon>0$ there exists $a \delta=\delta(\varepsilon, m, n)>0$ such that if $f$ : $M \rightarrow \mathbf{R}^{m} \times F \times \Delta^{n}$ and $f^{\prime}: M \rightarrow \mathbf{R}^{m} \times F \times \Delta^{n}$ are $f . p \cdot p^{-1}(\delta)$-equivalences and $\tau(f)$ and $\tau\left(f^{\prime}\right)$ are defined, then $\tau(f)=\tau\left(f^{\prime}\right)$ if and only if there is an $f$.p. homeomorphism $h: M \rightarrow M^{\prime}$ such that $h\left|\mathbf{R}^{m} \times F \times \partial \Delta^{n}=\mathrm{id}, f^{\prime} h\right| \rho^{-1}\left(\partial \Delta^{n}\right)=f \mid \rho^{-1}\left(\partial \Delta^{n}\right)$, and $f^{\prime} h$ is $f . p$. $p^{-1}(\varepsilon)$-homotopic rel $\rho^{-1}\left(\partial \Delta^{n}\right)$ to $f$.

Proof. The proof is completely analogous to the proof of Proposition 3.6. One simply keeps track of the control at each step of the proof. The key observation is that if $\tau(f)$ is defined to be $[\tilde{f}]$, then one may assume that $f$ is f.p. $p^{-1}(\varepsilon)$-homotopic rel $\rho^{-1}\left(\partial \Delta^{n}\right)$ to $\tilde{f}$.

4. Proof of Theorem 3. The purpose of this section is to compute $\pi_{0} \mathscr{W} h\left(\mathbf{R}^{m} \times F\right)$ by reinterpreting a result of Chapman [8, Theorem 2]. It is first shown how to define addition so that $\pi_{0} \mathscr{W} h\left(\mathbf{R}^{m} \times F\right)$ becomes an abelian group.

Let $f: M \rightarrow \mathbf{R}^{m} \times F$ and $f^{\prime}: M^{\prime} \rightarrow \mathbf{R}^{m} \times F$ represent elements [ $\left.f\right]$ and $\left[f^{\prime}\right]$ of $\pi_{0} \mathscr{W} h\left(\mathbf{R}^{m} \times F\right)$. Let $N$ be the $Q$-manifold obtained by gluing $M$ and $M^{\prime}$ together along their common copy of $\mathbf{R}^{m} \times F$. Define $g: N \rightarrow \mathbf{R}^{m} \times F$ by $g \mid M=f$ and $g \mid M^{\prime}=f^{\prime}$. Of course $\mathbf{R}^{m} \times F$ is not a $Z$-set in $N$, but $g$ is a $p^{-1}(\varepsilon)$-sdr for every $\varepsilon>0$. Define $[f]+\left[f^{\prime}\right]$ to be $\tau(g)$ in $\pi_{0} \mathscr{W} h\left(\mathbf{R}^{m} \times F\right)$. That this addition operation is well defined follows directly from Propositions 3.4 and 3.6. Existence of inverses for this addition follows from the usual geometric construction [13, p. 21] with control [8, p. 320]. Alternatively, one may note that in what follows we show that $\pi_{0} \mathscr{W} h\left(\mathbf{R}^{m} \times F\right)$ is monoid-isomorphic to an abelian group. Inverses then exist automatically and $\pi_{0} \mathscr{W} h\left(\mathbf{R}^{m} \times F\right)$ is an abelian group. Note that the identity element is represented by the projection map $\mathbf{R}^{m} \times F \times[0,1] \rightarrow \mathbf{R}^{m} \times F$.

In [8] Chapman defines $\mathscr{S}_{b}\left(\mathbf{R}^{m} \times F\right)$ to be the set of equivalence classes of the form [ $f$ ], where $f: M \rightarrow \mathbf{R}^{m} \times F$ is a bounded homotopy equivalence and $M$ is a $Q$-manifold. Another such map, $f^{\prime}: M^{\prime} \rightarrow \mathbf{R}^{m} \times F$, is defined to be equivalent to $f$ provided that there is a homeomorphism $h: M \rightarrow M^{\prime}$ for which $f^{\prime} h$ is boundedly homotopic to $f$.

Proposition 4.1. There is a one-to-one correspondence $\gamma: \pi_{0} \mathscr{W} h\left(\mathbf{R}^{m} \times F\right) \rightarrow$ $\mathscr{S}_{b}\left(\mathbf{R}^{m} \times F\right)$.

PROoF. If $f$ represents a class [ $f]$ in $\pi_{0} \mathscr{W} h\left(\mathbf{R}^{m} \times F\right)$, then $f$ represents a class in $\mathscr{S}_{b}\left(\mathbf{R}^{m} \times F\right)$, also denoted $[f]$. Define $\gamma([f])=[f]$. It follows from Proposition 3.4 that $\gamma$ is well defined. Now define $\tau: \mathscr{S}_{b}\left(\mathbf{R}^{m} \times F\right) \rightarrow \pi_{0} \mathscr{W} h\left(\mathbf{R}^{m} \times F\right)$ by $\tau([f])=$ $\tau(f)$, the torsion of $f$ in $\pi_{0} \mathscr{W} h\left(\mathbf{R}^{m} \times F\right)$. It follows from Proposition 3.6 that $\tau$ is well defined.

It is clear that $\tau \circ \gamma=\mathrm{id}$. And it follows from the definition of torsion that $\gamma \circ \tau=\mathrm{id}$. Thus, $\gamma$ is a one-to-one correspondence.

Let $\tilde{K}_{i}(F)$ denote $\mathrm{Wh}\left(\mathbf{Z} \pi_{1} F\right)$ for $i=1, \tilde{K}_{0}\left(\mathbf{Z} \pi_{1} F\right)$ for $i=0$, and $K_{i}\left(\mathbf{Z} \pi_{1} F\right)$ for $i<0$. In [8, §8] Chapman defines a one-to-one correspondence $\sigma_{*}: \mathscr{S}_{b}\left(\mathbf{R}^{m} \times F\right) \rightarrow$ $\tilde{K}_{1-m}(F)$. 
PROPOSITION 4.2. The composition $\sigma_{*} \gamma: \pi_{0} \mathscr{W} h\left(\mathbf{R}^{m} \times F\right) \rightarrow \tilde{K}_{1-m}(F)$ is a group isomorphism.

Proof. It suffices to show that that $\sigma_{*} \gamma$ is a group homomorphism. For this one needs to examine Chapman's definition of $\sigma_{*}$. Let $\left[f_{i}\right]$ be an element of

$$
\pi_{0} \mathscr{W} h\left(\mathbf{R}^{m} \times F\right) \text { for } i=1,2 .
$$

By a wrapping-up procedure Chapman constructs a homotopy equivalence $\tilde{f}_{i}$ : $\tilde{M}_{i} \rightarrow T^{m} \times F$ with small control in the $T^{m}$-direction for $i=1,2$. Using a relative version of this wrapping-up procedure as developed in [23] one may assume that $\tilde{M}_{i}$ contains $T^{m} \times F$ as a $Z$-set and $\tilde{f}_{i} \mid T^{m} \times F=$ id for $i=1,2$. Chapman shows that $\tau\left(\tilde{f}_{i}\right)$ lies in the subgroup $\mathrm{Wh}(F) \oplus \sum_{i=1}^{m}\left(\begin{array}{c}m \\ i\end{array}\right) \tilde{K}_{1-i}(F)$ of $\mathrm{Wh}\left(T^{m} \times F\right)$ and defines $\sigma_{*}\left(\left[\tilde{f}_{i}\right]\right)$ to be the component of $\tau\left(\tilde{f}_{i}\right)$ in $\tilde{K}_{1-m}(F)$. By our choice of $\tilde{M}_{i}$ we see that $\sigma_{*} \gamma\left(\left[f_{1}\right]+\left[f_{2}\right]\right)=\sigma_{*} \gamma\left(\left[f_{1}\right]\right)+\sigma_{*} \gamma\left(\left[f_{2}\right]\right)$.

5. Proof of Theorem 1. In this section we define an isomorphism

$$
\alpha: \pi_{n} \mathscr{W} h\left(\mathbf{R}^{m} \times F\right) \rightarrow \pi_{n-1} \mathscr{C}_{b}\left(\mathbf{R}^{m} \times F\right) .
$$

The key ingredient which allows us to construct bounded concordances from elements of $\pi_{n} \mathscr{W} h\left(\mathbf{R}^{m} \times F\right)$ is Theorem 2.3.

To define $\alpha$ let $f: M \rightarrow \mathbf{R}^{m} \times F \times \Delta^{n}$ represent an element $[f]$ of $\pi_{n} \mathscr{W} h\left(\mathbf{R}^{m} \times F\right)$. By Lemma 3.3 there is an f.p. homeomorphism $j: \mathbf{R}^{m} \times F \times[0,1] \times \partial \Delta^{n} \rightarrow \rho^{-1}\left(\partial \Delta^{n}\right)$ such that $j \mid \mathbf{R}^{m} \times F \times\{0\} \times \partial \Delta^{n}=$ id and $f j$ is projection. The following lemma establishes a particularly useful trivialization of $\rho$.

LEMMA 5.1. There exists an f.p. homeomorphism $h: \mathbf{R}^{m} \times F \times[0,1] \times \Delta^{n} \rightarrow M$ such that $h \mid \mathbf{R}^{m} \times F \times\{0\} \times \Delta^{n}=\mathrm{id}$ and $h\left|\mathbf{R}^{m} \times F \times[0,1] \times \partial_{n} \Delta^{n}=j\right|$.

Proof. Since $\rho: M \rightarrow \Delta^{n}$ is a trivial bundle, one can construct an f.p. homeomorphism $h^{\prime}: \mathbf{R}^{m} \times F \times[0,1] \times \Delta^{n} \rightarrow M$ such that $h^{\prime}\left|\mathbf{R}^{m} \times F \times[0,1] \times \partial_{n} \Delta^{n}=j\right|$. It follows that $h^{\prime} \mid \mathbf{R}^{m} \times F \times\{0\} \times \Delta^{n}$ is f.p. homotopic rel $\mathbf{R}^{m} \times F \times\{0\} \times \partial_{n} \Delta^{n}$ to the identity. The existence of $h$ now follows from sliced $Z$-set unknotting.

With the homeomorphisms $j$ and $h$ in hand, consider the $n$-parameter family of approximate fibrations $p f h: \mathbf{R}^{m} \times F \times[0,1] \times \Delta^{n} \rightarrow \mathbf{R}^{m} \times \Delta^{n}$. Note that

$$
p f h \mid\left(\mathbf{R}^{m} \times F \times[0,1] \times \partial_{n} \Delta^{n}\right) \cup\left(\mathbf{R}^{m} \times F \times\{0\} \times \Delta^{n}\right)
$$

is projection. It follows from Theorem 2.3 that there is an f.p. homeomorphism $H$ : $\mathbf{R}^{m} \times F \times[0,1] \times \Delta^{n} \rightarrow \mathbf{R}^{m} \times F \times[0,1] \times \Delta^{n}$ such that $H \mid\left(\mathbf{R}^{m} \times F \times[0,1] \times \partial_{n} \Delta^{n}\right)$ $\cup\left(\mathbf{R} \times F \times\{0\} \times \Delta^{n}\right)=$ id and the composition $p f h H$ is $\varepsilon$-close to projection. Here, $\varepsilon$ is chosen small enough for the proof of Proposition 5.2 to work.

Let $J^{n-1}$ denote the $(n-1)$-cell $\partial \Delta^{n} \backslash \operatorname{int}\left(\partial_{n} \Delta^{n}\right)$. Consider the composition

$$
\begin{aligned}
\tilde{h}: \mathbf{R}^{m} \times F \times[0,1] \times J^{n-1} & \stackrel{H \mid}{\rightarrow} \mathbf{R}^{m} \times F \times[0,1] \times J^{n-1} \\
& \stackrel{h \mid}{\rightarrow} \rho^{-1}\left(J^{n-1}\right) \stackrel{(j)^{-1}}{\rightarrow} \mathbf{R}^{m} \times F \times[0,1] \times J^{n-1} .
\end{aligned}
$$


If one considers the $(n-1)$-simplices of $\mathscr{C}_{b}\left(\mathbf{R}^{m} \times F\right)$ to be parametrized by $J^{n-1}$ (instead of $\left.\Delta^{n-1}\right)$, then it follows that $\tilde{h}$ determines a class $[\tilde{h}]$ in $\pi_{n-1} \mathscr{C}_{b}\left(\mathbf{R}^{m} \times F\right)$. Define $\alpha([f])=[\tilde{h}]$.

Proposition 5.2. $\alpha$ is well defined.

Proof. If $f: M \rightarrow \mathbf{R}^{m} \times F \times \Delta^{n}$ represents [ $f$ ] in $\pi_{n} \mathscr{W} h\left(\mathbf{R}^{m} \times F\right)$, we first show that the definition of $\alpha([f])$ is independent of the choices for the homeomorphisms $j$, $h$ and $H$. So suppose that alternative choices $j^{\prime}, h^{\prime}$ and $H^{\prime}$ have been made.

Consider the f.p. homeomorphism $\left(j^{\prime}\right)^{-1} j: \mathbf{R}^{m} \times F \times[0,1] \times \partial \Delta^{n} \rightarrow \mathbf{R}^{m} \times F \times$ $[0,1] \times \partial \Delta^{n}$. Note that $\left(j^{\prime}\right)^{-1} j \mid \mathbf{R}^{m} \times F \times\{0\} \times \partial \Delta^{n}=$ id and that $\left(j^{\prime}\right)^{-1} j$ affects only the $[0,1]$-coordinate of any point. Then $\left(j^{\prime}\right)^{-1} j$ can be thought of as defining a parametrized family of homeomorphisms on $[0,1]$ which are fixed on $\{0,1\}$. It therefore follows from an Alexander trick that there is a homeomorphism

$$
\hat{j}: \mathbf{R}^{m} \times F \times[0,1] \times \partial \Delta^{n} \times I \rightarrow \mathbf{R}^{m} \times F \times[0,1] \times \partial \Delta^{n} \times I
$$

such that:

(i) $\hat{j}$ affects only the $[0,1]$-coordinate of any point;

(ii) $\hat{j} \mid \mathbf{R}^{m} \times F \times\{0\} \times \partial \Delta^{n} \times I=$ id;

(iii) $\hat{j} \mid \mathbf{R}^{m} \times F \times[0,1] \times \partial \Delta^{n} \times\{0\}=\left(j^{\prime}\right)^{-1} j$;

(iv) $\hat{j} \mid \mathbf{R}^{m} \times F \times[0,1] \times \partial \Delta^{n} \times\{1\}=$ id.

Define $\tilde{j}: \mathbf{R}^{m} \times F \times[0,1] \times \partial \Delta^{n} \times I \rightarrow \rho^{-1}\left(\partial \Delta^{n}\right) \times I$ by setting $\tilde{j}=\left(j^{\prime} \times\right.$ id $) \hat{j}$.

Since $\partial_{n} \Delta^{n}$ is a strong deformation retract of $\Delta^{n}$, there is a homotopy $r_{u}: \Delta^{n} \rightarrow \Delta^{n}$, $0 \leqslant u \leqslant 1$, such that $r_{0}=\mathrm{id},\left.r_{u}\right|_{n} \Delta^{n}=\mathrm{id}$, and $r_{1}\left(\Delta^{n}\right)=\partial_{n} \Delta^{n}$. Define a homeomorphism

$$
\hat{h}: \mathbf{R}^{m} \times F \times[0,1] \times \Delta^{n} \times I \rightarrow \mathbf{R}^{m} \times F \times[0,1] \times \Delta^{n} \times I
$$

by setting

$$
\hat{h}(x, f, s, t, u)=\left(\operatorname{prh}^{-1} h^{\prime}\left(x, f, s, r_{1-u}(t)\right), t, u\right),
$$

where pr denotes projection from $\mathbf{R}^{m} \times F \times[0,1] \times \Delta^{n}$ to $\mathbf{R}^{m} \times F \times[0,1]$. Note that $\hat{h}$ has the following properties:

(i) $\hat{h} \mid \mathbf{R}^{m} \times F \times\{0\} \times \Delta^{n} \times I=$ id;

(ii) $\hat{h} \mid \mathbf{R}^{m} \times F \times[0,1] \times \Delta^{n} \times\{0\}$ affects only the [0,1]-coordinate of any point;

(iii) $\hat{h} \mid \mathbf{R}^{m} \times F \times[0,1] \times \Delta^{n} \times\{1\}=h^{-1} h^{\prime}$;

(iv) $\hat{h}\left|\mathbf{R}^{m} \times F \times[0,1] \times \partial_{n} \Delta^{n} \times I=j^{-1} j^{\prime}\right| \times$ id.

Now $\hat{h}$ extends to a homeomorphism $\hat{h}: \mathbf{R}^{m} \times F \times[0,1] \times \Delta^{n} \times[-1,1] \rightarrow \mathbf{R}^{m} \times F$ $\times[0,1] \times \Delta^{n} \times[-1,1]$ such that:

(i) $\hat{h} \mid \mathbf{R}^{m} \times F \times\{0\} \times \Delta^{n} \times[-1,1]=\mathrm{id}$;

(ii) $\hat{h} \mid \mathbf{R}^{m} \times F \times[0,1] \times \Delta^{n} \times[-1,0]$ affects only the [0,1]-coordinate of any point;

(iii) $\hat{h} \mid \mathbf{R}^{m} \times F \times[0,1] \times \Delta^{n} \times\{-1\}=$ id.

To return to the parameter space $I$ instead of $[-1,1]$, define

$$
h^{*}: \mathbf{R}^{m} \times F \times[0,1] \times \Delta^{n} \times I \rightarrow \mathbf{R}^{m} \times F \times[0,1] \times \Delta^{n} \times I
$$

by setting

$$
h^{*}(x, f, s, t, u)=\hat{\hat{h}}(x, f, s, t, 2 u-1) \text {. }
$$


Consider the parametrized family of approximate fibrations

$q=p(f \times$ id $)(h \times$ id $) h^{*}(H \times$ id $): \mathbf{R}^{m} \times F \times[0,1] \times \Delta^{n} \times I \rightarrow \mathbf{R}^{m} \times \Delta^{n} \times I$.

Note that:

(i) $q \mid \mathbf{R}^{m} \times F \times\{0\} \times \Delta^{n} \times I$ is projection;

(ii) $q \mid \mathbf{R}^{m} \times F \times[0,1] \times \Delta^{n} \times\{0\}=p f h H$;

(iii) $q \mid \mathbf{R}^{m} \times F \times[0,1] \times \Delta^{n} \times\{1\}=p f h^{\prime} H$;

(iv) $q \mid \mathbf{R}^{m} \times F \times[0,1] \times \partial_{n} \Delta^{n} \times I$ is projection.

By Theorem 2.3 there is an f.p. homeomorphism $\tilde{H}: \mathbf{R}^{m} \times F \times[0,1] \times \Delta^{n} \times I \rightarrow$ $\mathbf{R}^{m} \times F \times[0,1] \times \Delta^{n} \times I$ such that:

(i) $q \tilde{H}$ is close to projection;

(ii) $\tilde{H} \mid \mathbf{R}^{m} \times F \times\{0\} \times \Delta^{n} \times I=$ id;

(iii) $\tilde{H} \mid \mathbf{R}^{m} \times F \times[0,1] \times \partial_{n} \Delta^{n} \times I=$ id;

(iv) $\tilde{H} \mid \mathbf{R}^{m} \times F \times[0,1] \times \Delta^{n} \times\{0\}=$ id;

(v) $\tilde{H} \mid \mathbf{R}^{m} \times F \times[0,1] \times \Delta^{n} \times\{1\}=H^{-1} H^{\prime}$.

(The reader should beware that we must "reparametrize" the $(n+1)$-cell $\Delta^{n} \times I$ so that the wording of Theorem 2.3 will apply.)

Now consider the composition

$$
\begin{aligned}
& G: \mathbf{R}^{m} \times F \times[0,1] \times J^{n-1} \times I \stackrel{\tilde{H} \mid}{\rightarrow} \mathbf{R}^{m} \times F \times[0,1] \times J^{n-1} \times I \\
& \stackrel{(h \times \mathrm{id}) h^{*}(H \times \mathrm{id}) \mid}{\rightarrow} \rho^{-1}\left(J^{n-1}\right) \times I \stackrel{(\tilde{j})^{-1}}{\rightarrow} \mathbf{R}^{m} \times F \times[0,1] \times J^{n-1} \times I .
\end{aligned}
$$

Then $G$ is a bounded concordance (parametrized over $J^{n-1} \times I$ ) such that:

(i) $G \mid \mathbf{R}^{m} \times F \times[0,1] \times J^{n-1} \times\{0\}=\tilde{h}$;

(ii) $G \mid \mathbf{R}^{m} \times F \times[0,1] \times J^{n-1} \times\{1\}=\tilde{h}^{\prime}$;

(iii) $G \mid \mathbf{R}^{m} \times F \times[0,1] \times \partial J^{n-1} \times I$ affects only the [0,1]-coordinate of any point.

Here $\tilde{h}$ and $\tilde{h}^{\prime}$ are the two concordances which determine the definition of $\alpha([f])$ depending on whether $j, h$ and $H$ or $j^{\prime}, h^{\prime}$ and $H^{\prime}$ are used.

Finally, one modifies $G$ (by an Alexander trick again) to get a bounded concordance

$$
\tilde{G}: \mathbf{R}^{m} \times F \times[0,1] \times J^{n-1} \times I \rightarrow \mathbf{R}^{m} \times F \times[0,1] \times J^{n-1} \times I
$$

parametrized over $J^{n-1} \times I$ such that:

(i) $\tilde{G}\left|\mathbf{R}^{m} \times F \times[0,1] \times J^{n-1} \times \partial I=G\right|$;

(ii) $\tilde{G} \mid \mathbf{R}^{m} \times F \times[0,1] \times \partial J^{n-1} \times I=$ id.

Then $\tilde{G}$ shows that $[\tilde{h}]=\left[\tilde{h}^{\prime}\right]$ in $\pi_{n-1} \mathscr{C}_{b}\left(\mathbf{R}^{m} \times F\right)$.

To complete the proof of the proposition we must show that the definition of $\alpha([f])$ does not depend on the choice of a representative of the equivalence class $[f]$. To this end let $f^{\prime}: M^{\prime} \rightarrow \mathbf{R}^{m} \times F \times \Delta^{n}$ be a map such that $[f]=\left[f^{\prime}\right]$ in

$$
\pi_{n} \mathscr{W h}\left(\mathbf{R}^{m} \times F\right)
$$

By Proposition 3.4 there is an f.p. homeomorphism $g: M \rightarrow M^{\prime}$ such that $g \mid \mathbf{R}^{m} \times F$ $\times \Delta^{n}=\mathrm{id}, f^{\prime} g\left|\rho^{-1}\left(\partial \Delta^{n}\right)=f\right|$, and $f^{\prime} g$ is close to $f$. It follows that if $j, h$ and $H$ are homeomorphisms used to define $\alpha([f])$, then $g j, g h$ and $H$ are acceptable choices of 
homeomorphisms which can be used to define $\alpha\left(\left[f^{\prime}\right]\right)=\left[\tilde{h}^{\prime}\right]$, where

$$
\begin{aligned}
\tilde{h}^{\prime}: \mathbf{R}^{m} \times F \times[0,1] \times J^{n-1} & \stackrel{H \mid}{\rightarrow} \mathbf{R}^{m} \times F \times[0,1] \times J^{n-1} \\
& \stackrel{(g h) \mid}{\rightarrow}\left(\rho^{\prime}\right)^{-1}\left(J^{n-1}\right) \stackrel{(g j)^{-1}}{\rightarrow} \mathbf{R}^{m} \times F \times[0,1] \times J^{n-1} .
\end{aligned}
$$

Now note that $\tilde{h}^{\prime}=\tilde{h}$, where $[\tilde{h}]=\alpha([f])$.

PROPOSITION 5.3. $\alpha$ is a group homomorphism.

Proof. We only treat the case $n=1$, where $\alpha: \pi_{1} \mathscr{W} h\left(\mathbf{R}^{m} \times F\right) \rightarrow \pi_{0} \mathscr{C}_{b}\left(\mathbf{R}^{m} \times F\right)$. The case $n>1$ is similar but easier; it follows more directly from the definitions. Recall that the group operation on $\pi_{0} \mathscr{C}_{b}\left(\mathbf{R}^{m} \times F\right)$ is induced by the composition of concordances.

Let $[f],\left[f^{\prime}\right] \in \pi_{1} \mathscr{W} h\left(\mathbf{R}^{m} \times F\right)$. The first step is to define explicitly a map $\tilde{f}$ so that $[f]+\left[f^{\prime}\right]=[\tilde{f}]$ in $\pi_{1} \mathscr{W} h\left(\mathbf{R}^{m} \times F\right)$. We may assume that $f, f^{\prime}: \mathbf{R}^{m} \times F \times[0,1] \times \Delta^{1}$ $\rightarrow \mathbf{R}^{m} \times F \times \Delta^{1}$, that $f, f^{\prime} \mid \mathbf{R}^{m} \times F \times\{0\} \times \Delta^{1}=$ id, and that $f, f^{\prime} \mid \mathbf{R}^{m} \times F \times[0,1] \times$ $\partial_{1} \Delta^{1}$ are projections. Moreover, there are homeomorphisms $j, j^{\prime}: \mathbf{R}^{m} \times F \times[0,1] \rightarrow$ $\mathbf{R}^{m} \times F \times[0,1] \times \partial_{0} \Delta^{1}$ such that $j, j^{\prime} \mid \mathbf{R}^{m} \times F \times\{0\}=\mathrm{id}$ and $f j$ and $f^{\prime} j^{\prime}$ are projections. When convenient we will think of $j$ and $j^{\prime}$ as being defined on $\mathbf{R}^{m} \times F \times[0,1]$ $\times \partial_{0} \Delta^{1}$.

Let pr: $\mathbf{R}^{m} \times F \times \Delta^{1} \rightarrow \mathbf{R}^{m} \times F$ denote projection and define $\tilde{f}: \mathbf{R}^{m} \times F \times[0,1]$ $\times \Delta^{1} \rightarrow \mathbf{R}^{m} \times F \times \Delta^{1}$ by

$$
\tilde{f}(x, f, s, t)= \begin{cases}(\operatorname{pr} f(x, f, s, 2 t), t) & \text { for } 0 \leqslant t \leqslant \frac{1}{2} \\ \left(\operatorname{pr} f^{\prime}\left(j^{-1}(x, f, s, 1), 2 t-1\right), t\right) & \text { for } \frac{1}{2} \leqslant t \leqslant 1\end{cases}
$$

Here $\Delta^{1}=[0,1]$. Then $[f]+\left[f^{\prime}\right]=[\tilde{f}]$.

Now $\alpha([f])$ and $\alpha\left(\left[f^{\prime}\right]\right)$ are defined by finding f.p. homeomorphisms $H, H^{\prime}$ : $\mathbf{R}^{m} \times F \times[0,1] \times \Delta^{1} \rightarrow \mathbf{R}^{m} \times F \times[0,1] \times \Delta^{1}$ such that $H, H^{\prime} \mid \mathbf{R}^{m} \times F \times\{0\} \times \Delta^{1}=$ id, $H, H^{\prime} \mid \mathbf{R}^{m} \times F \times[0,1] \times \partial_{1} \Delta^{1}=$ id, and $p f H$ and $p f^{\prime} H^{\prime}$ are close to projection. Then $\alpha([f])=[\tilde{h}]$, where $\tilde{h}$ is the composition

$$
\begin{aligned}
\tilde{h}: \mathbf{R}^{m} \times F \times[0,1] \times \partial_{0} \Delta^{1} \stackrel{H \mid}{\rightarrow} \mathbf{R}^{m} \times F \times[0,1] \times \partial_{0} \Delta^{1} \\
\stackrel{j^{-1}}{\rightarrow} \mathbf{R}^{m} \times F \times[0,1] \times \partial_{0} \Delta^{1} .
\end{aligned}
$$

Similarly, $\alpha\left(\left[f^{\prime}\right]\right)=\left[\tilde{h}^{\prime}\right]$.

Let $\widehat{\text { pr: }} \mathbf{R}^{m} \times F \times[0,1] \times \Delta^{1} \rightarrow \mathbf{R}^{m} \times F \times[0,1]$ denote projection and define $\tilde{H}$ : $\mathbf{R}^{m} \times F \times[0,1] \times \Delta^{1} \rightarrow \mathbf{R}^{m} \times F \times[0,1] \times \Delta^{1}$ by

$$
\tilde{H}(x, f, s, t)= \begin{cases}(\widehat{\operatorname{pr}} H(x, f, s, 2 t), t) & \text { for } 0 \leqslant t \leqslant \frac{1}{2} \\ \left(\hat{\operatorname{pr}} H^{\prime}\left(j^{-1} H(x, f, s, 1), 2 t-1\right), t\right) & \text { for } \frac{1}{2} \leqslant t \leqslant 1 .\end{cases}
$$


Then $p \tilde{f} \tilde{H}$ is close to projection and $\alpha([\tilde{f}])=[\hat{h}]$, where $\hat{h}$ is the composition

$$
\begin{aligned}
\hat{h}: \mathbf{R}^{m} \times F \times[0,1] \times \partial_{0} \Delta^{1} & \stackrel{\tilde{H} \mid}{\rightarrow} \mathbf{R}^{m} \times F \times[0,1] \times \partial_{0} \Delta^{1} \\
\stackrel{\left(j j^{\prime}\right)^{-1}}{\rightarrow} & \mathbf{R}^{m} \times F \times[0,1] \times \partial_{0} \Delta^{1} .
\end{aligned}
$$

To complete the proof observe that $\hat{h}=\tilde{h}^{\prime} \tilde{h}$.

Proposition 5.4. $\alpha$ is injective.

Proof. Let $[f] \in \pi_{n} \mathscr{W} h\left(\mathbf{R}^{m} \times F\right)$ such that $\alpha([f])=0$. To define $\alpha([f])$ we are given homeomorphisms $j, h$ and $H$ as above. Then $\alpha([f])=[\tilde{h}]$, where $\tilde{h}=j^{-1} h H \mid \mathbf{R}^{m}$ $\times F \times[0,1] \times J^{n-1}$. If $[\tilde{h}]=0$ in $\pi_{n-1} \mathscr{C}_{b}\left(\mathbf{R}^{m} \times F\right)$, there exists a bounded homeomorphism

$$
G: \mathbf{R}^{m} \times F \times[0,1] \times J^{n-1} \times I \rightarrow \mathbf{R}^{m} \times F \times[0,1] \times J^{n-1} \times I
$$

which is f.p. over $J^{n-1} \times I, G \mid \mathbf{R}^{m} \times F \times[0,1] \times J^{n-1} \times\{0\}=\tilde{h}$, and $G$ is the identity on $\mathbf{R}^{m} \times F \times\{0\} \times J^{n-1} \times I$, on $\mathbf{R}^{m} \times F \times[0,1] \times \partial J^{n-1} \times I$, and on $\mathbf{R}^{m} \times F \times[0,1] \times J^{n-1} \times\{1\}$.

Let

$$
r:[0,1] \times \Delta^{n} \times I \rightarrow\left(\{0\} \times \Delta^{n} \times I\right) \cup\left([0,1] \times \partial\left(\Delta^{n} \times I\right)\right)
$$

be a retraction. Let pr: $\mathbf{R}^{m} \times F \times[0,1] \times \Delta^{n} \times I \rightarrow \mathbf{R}^{m} \times F \times \Delta^{n} \times I$ denote projection. Define $D: \mathbf{R}^{m} \times F \times[0,1] \times \Delta^{n} \times I \rightarrow \mathbf{R}^{m} \times F \times \Delta^{n} \times I$ by

$$
D(x, f, s, t, u)=\left\{\begin{aligned}
&(x, f, t, u) \quad \text { if } r(s, t, u) \in\left(\{0\} \times \Delta^{n} \times I\right) \\
& \cup\left([0,1] \times \Delta^{n} \times\{1\}\right) \cup\left([0,1] \times \partial_{n} \Delta^{n} \times I\right), \\
& \operatorname{pr} G(x, f, r(s, t, u)) \text { if } r(s, t, u) \in[0,1] \times J^{n-1} \times I, \\
&(f h H(x, f, r(s, t, u)), 1) \quad \text { if } r(s, t, u) \in[0,1] \times \Delta^{n} \times\{1\} .
\end{aligned}\right.
$$

Note that $p D$ is boundedly close to projection. Thus Proposition 3.1 implies that there is an f.p. map $\tilde{D}: \mathbf{R}^{m} \times F \times[0,1] \times \Delta^{n} \times I \rightarrow \mathbf{R}^{m} \times F \times \Delta^{n} \times I$ such that $\tilde{D}\left|\mathbf{R}^{m} \times F \times\{0\} \times \Delta^{n} \times I=\mathrm{id}, \tilde{D}\right| \mathbf{R}^{m} \times F \times[0,1] \times \partial\left(\Delta^{n} \times I\right)=\mathrm{id}$, and $p \tilde{D}$ is an approximate fibration.

Then $\tilde{D}$ provides a homotopy showing that $[f h H]=0$ in $\pi_{n} \mathscr{W} h\left(\mathbf{R}^{m} \times F\right)$. Since $[f]=[f h H]$ we have shown $\operatorname{ker}(\alpha)=0$.

Proposition 5.5. $\alpha$ is surjective.

Proof. Let $[G] \in \pi_{n-1} \mathscr{C}_{b}\left(\mathbf{R}^{m} \times F\right)$. Then $G$ is an f.p. bounded homeomorphism

$$
G: \mathbf{R}^{m} \times F \times[0,1] \times \Delta^{n-1} \rightarrow \mathbf{R}^{m} \times F \times[0,1] \times \Delta^{n-1}
$$

such that

$$
G \mid\left(\mathbf{R}^{m} \times F \times\{0\} \times \Delta^{n-1}\right) \cup\left(\mathbf{R}^{m} \times F \times[0,1] \times \partial \Delta^{n-1}\right)=\mathrm{id} .
$$

The straight line homotopy from the projection $\mathbf{R}^{m} \times F \times[0,1] \times \Delta^{n-1} \rightarrow \mathbf{R}^{m} \times$ $\Delta^{n-1}$ to $p G$ is bounded, f.p., and $\operatorname{rel}\left(\mathbf{R}^{m} \times F \times\{0\} \times \Delta^{n-1}\right) \cup\left(\mathbf{R}^{m} \times F \times[0,1] \times\right.$ $\left.\partial \Delta^{n-1}\right)$. This homotopy induces an f.p. map $g: \mathbf{R}^{m} \times F \times[0,1] \times \Delta^{n-1} \times I \rightarrow \mathbf{R}^{m} \times$ $\Delta^{n-1} \times I$ such that:

(i) $g \mid \mathbf{R}^{m} \times F \times\{0\} \times \Delta^{n-1} \times I=\mathrm{id}$; 
(ii) $g \mid\left(\mathbf{R}^{m} \times F \times[0,1] \times \partial \Delta^{n-1} \times I\right) \cup\left(\mathbf{R}^{m} \times F \times[0,1] \times \Delta^{n-1} \times\{0\}\right)$ is projection;

(iii) $g \mid \mathbf{R}^{m} \times F \times[0,1] \times \Delta^{n-1} \times\{1\}=p G$;

(iv) $g$ is boundedly close to projection.

As in Proposition 5.4, using the fact that

$$
\left(\{0\} \times \Delta^{n-1} \times I\right) \cup\left([0,1] \times \partial\left(\Delta^{n-1} \times I\right)\right)
$$

is a retract of $[0,1] \times \Delta^{n-1} \times I$, we can find an f.p. map $\hat{g}: \mathbf{R}^{m} \times F \times[0,1] \times \Delta^{n-1}$ $\times I \rightarrow \mathbf{R}^{m} \times F \times \Delta^{n-1} \times I$ such that:

(i) $\hat{g} \mid \mathbf{R}^{m} \times F \times\{0\} \times \Delta^{n-1} \times I=$ id;

(ii) $\hat{g} \mid\left(\mathbf{R}^{m} \times F \times[0,1] \times \partial \Delta^{n-1} \times I\right) \cup\left(\mathbf{R}^{m} \times F \times[0,1] \times \partial \Delta^{n-1} \times\{0\}\right)$ is projection;

(iii) $\hat{g} \mid \mathbf{R}^{m} \times F \times[0,1] \times \Delta^{n-1} \times\{1\}=\operatorname{pr} G$ (where pr: $\mathbf{R}^{m} \times F \times[0,1] \times \Delta^{n-1} \rightarrow$ $\mathbf{R}^{m} \times F \times \Delta^{n-1}$ is projection);

(iv) $p \hat{g}=g$.

By Proposition 3.2 there is an f.p. map $\tilde{g}: \mathbf{R}^{m} \times F \times[0,1] \times \Delta^{n-1} \times I \rightarrow \mathbf{R}^{m} \times F$ $\times \Delta^{n-1} \times I$ such that $\tilde{g}\left|\mathbf{R}^{m} \times F \times\{0\} \times \Delta^{n-1} \times I=\mathrm{id}, \quad \tilde{g}\right| \mathbf{R}^{m} \times F \times[0,1] \times$ $\partial\left(\Delta^{n-1} \times I\right)=\hat{g} \mid$, and $p \tilde{g}$ is an approximate fibration.

Identify $\Delta^{n}$ with $\Delta^{n-1} \times I$ in such a way that $\partial_{n} \Delta^{n}$ is identified with $\left(\Delta^{n-1} \times\{0\}\right)$ $\cup\left(\partial \Delta^{n-1} \times I\right)$. Then $\tilde{g}$ determines a class $[\tilde{g}]$ in $\pi_{n} \mathscr{W} h\left(\mathbf{R}^{m} \times F\right)$ and one checks that $\alpha([\tilde{g}])=[G]$.

6. Proof of Theorem 2. In this section we define an isomorphism

$$
\beta: \pi_{n} \mathscr{C}_{b}\left(\mathbf{R}^{m+1} \times F\right) \rightarrow \pi_{n} \mathscr{W} h\left(\mathbf{R}^{m} \times F\right) .
$$

The construction is similar to that in $[1, \S 8]$.

In order to define $\beta$, let [ $h]$ be an element of $\pi_{n} \mathscr{C}_{b}\left(\mathbf{R}^{m+1} \times F\right)$. Thus $h$ is an f.p. bounded homeomorphism $h: \mathbf{R}^{m+1} \times F \times[0,1] \times \Delta^{n} \rightarrow \mathbf{R}^{m+1} \times F \times[0,1] \times \Delta^{n}$ such that $h \mid\left(\mathbf{R}^{m+1} \times F \times\{0\} \times \Delta^{n}\right) \cup\left(\mathbf{R}^{m+1} \times F \times[0,1] \times \partial \Delta^{n}\right)=$ id. Let $L$ be a positive number such that $p h$ is $(L / 4)$-close to projection. Write $\mathbf{R}^{m+1}$ as $\mathbf{R}^{m} \times \mathbf{R}$ and define $M$ to be

$$
h\left(\mathbf{R}^{m} \times(-\infty, L] \times F \times[0,1] \times \Delta^{n}\right) \backslash\left(\mathbf{R}^{m} \times(-\infty, 0) \times F \times[0,1] \times \Delta^{n}\right) .
$$

Since $M$ is a subset of $\mathbf{R}^{m+1} \times F \times[0,1] \times \Delta^{n}$, the projection to $\Delta^{n}$ restricts to a map $\rho: M \rightarrow \Delta^{n}$.

Note that $\mathbf{R}^{m} \times\{0\} \times F \times[0,1] \times \Delta^{n}$ is a sliced $Z$-set in $M$. Define $f: M \rightarrow \mathbf{R}^{m} \times$ $\{0\} \times F \times[0,1] \times \Delta^{n}$ to be the restriction of the projection. We now show that $f$ is an f.p. bounded sdr. Let $r: \mathbf{R}^{m+1} \times F \times[0,1] \times \Delta^{n} \rightarrow \mathbf{R}^{m} \times(-\infty, L] \times F \times[0,1] \times$ $\Delta^{n}$ be the obvious retraction which affects only the R-coordinate of any point. Let $s_{t}$ : $\mathbf{R}^{m+1} \times F \times[0,1] \times \Delta^{n} \rightarrow \mathbf{R}^{m+1} \times F \times[0,1] \times \Delta^{n}, 0 \leqslant t \leqslant 1$, be the homotopy such that $s_{t}$ multiplies the $\mathbf{R}$-coordinate of any point by $t$ and does not affect the other coordinates. (By the R-coordinate we mean the last coordinate of a point in $\mathbf{R}^{m+1}=\mathbf{R}^{m} \times \mathbf{R}$.) Finally, define $f_{t}: M \rightarrow M, 0 \leqslant t \leqslant 1$, by $f_{t}=h r h^{-1} s_{t}$. Then $f_{1}=\mathrm{id}$ and $f_{0}=f$. This homotopy shows that $f$ is an f.p. bounded sdr.

LEMMA 6.1. The map $\rho: M \rightarrow \Delta^{n}$ is a bundle with $Q$-manifold fibers. 
Proof. It is clear that $\rho$ is a submersion with noncompact $Q$-manifold fibers. There are two alternative ways to show that $\rho$ is, in fact, a bundle. The author's original method was to follow the proof of the Technical Bundle Theorem in [25], constructing by hand the radial engulfing isotopies. (It is necessary here to appeal to the $Q$-manifold versions of the submersion theorem and the isotopy extension theorem in [17 and 31].)

The following quicker method was pointed out by the referee. Note that there is an f.p. proper retraction $R: \mathbf{R}^{m} \times[0,2 L] \times F \times[0,1] \times \Delta^{n} \rightarrow M$ defined by suitably restricting $h r h^{-1}$. Thus, $\rho$ is a so-called proper fibration and one simply invokes Theorem 2 of $[10]$ to conclude that $\rho$ is a bundle.

Since $\rho^{-1}\left(\partial \Delta^{n}\right)=\mathbf{R}^{m} \times[0, L] \times F \times[0,1] \times \partial \Delta^{n}$ and $f \mid \rho^{-1}\left(\partial \Delta^{n}\right)$ is projection, we may take the torsion of $f$ to obtain an element $\tau(f)$ in $\pi_{n} \mathscr{W} h\left(\mathbf{R}^{m} \times F \times[0,1]\right)$. A homeomorphism $k: F \times[0,1] \rightarrow F$ induces an isomorphism

$$
k_{*}: \pi_{n} \mathscr{W} h\left(\mathbf{R}^{m} \times F \times[0,1]\right) \rightarrow \pi_{n} \mathscr{W} h\left(\mathbf{R}^{m} \times F\right) .
$$

Now define $\beta([h])=k_{*}(\tau(f))$.

Proposition 6.2. $\beta$ is well defined.

Proof. We need to show that $\beta([h])$ does not depend on the choices for $k, L$, or the concordance representing $[h]$. First, it is clear that $k_{*}(\tau(f))$ is independent of the homeomorphism $k: F \times[0,1] \rightarrow F$.

Second, suppose $L^{\prime}>L$ is another number. This yields

$$
M^{\prime}=h\left(\mathbf{R}^{m} \times\left(-\infty, L^{\prime}\right] \times F \times[0,1] \times \Delta^{n}\right) \backslash \mathbf{R}^{m} \times(-\infty, 0) \times F \times[0,1] \times \Delta^{n}
$$

and an f.p. bounded $\operatorname{sdr} f^{\prime}: M^{\prime} \rightarrow \mathbf{R}^{m} \times\{0\} \times F \times[0,1] \times \Delta^{n}$ which is the restriction of the projection. Let $\gamma: \mathbf{R}^{m} \times(-\infty, L] \times F \times[0,1] \times \Delta^{n} \rightarrow \mathbf{R}^{m} \times\left(-\infty, L^{\prime}\right]$ $\times F \times[0,1] \times \Delta^{n}$ be the homeomorphism induced by the homeomorphism $(-\infty, L]$ $\rightarrow\left(-\infty, L^{\prime}\right]$ which is the identity on $(-\infty, L / 2]$ and takes $[L / 2, L]$ linearly onto $\left[L / 2, L^{\prime}\right]$. Define a homeomorphism $\tilde{\gamma}: M \rightarrow M^{\prime}$ by $\tilde{\gamma}=h \gamma h^{-1} \mid M$. Then $\tilde{\gamma}$ satisfies the hypothesis of Proposition 3.6 showing $\tau(f)=\tau\left(f^{\prime}\right)$.

Finally, let $h^{\prime}$ be a concordance such that $\left[h^{\prime}\right]=[h]$ in $\pi_{n} \mathscr{C}_{b}\left(\mathbf{R}^{m+1} \times F\right)$. Then there is an f.p. bounded homeomorphism $H: \mathbf{R}^{m+1} \times F \times[0,1] \times \Delta^{n} \times I \rightarrow \mathbf{R}^{m+1}$ $\times F \times[0,1] \times \Delta^{n} \times I$ such that $H \mid\left(\mathbf{R}^{m+1} \times F \times\{0\} \times \Delta^{n} \times I\right) \cup\left(\mathbf{R}^{m+1} \times F \times\right.$ $\left.[0,1] \times \partial \Delta^{n} \times I\right)=$ id, $H \mid \mathbf{R}^{m+1} \times F \times[0,1] \times \Delta^{n} \times\{0\}=h$, and $H \mid \mathbf{R}^{m+1} \times F \times$ $[0,1] \times \Delta^{n} \times\{1\}=h^{\prime}$. Let $\tilde{L}>0$ be chosen such that $p H$ is $(\tilde{L} / 4)$-close to projection. Then as was done above we obtain

$$
\tilde{M}=H\left(\mathbf{R}^{m} \times(-\infty, \tilde{L}] \times F \times[0,1] \times \Delta^{n} \times I\right) \backslash\left(\mathbf{R}^{m} \times(-\infty, 0) \times F \times[0,1] \times \Delta^{n} \times I\right),
$$

a bundle $\tilde{\rho}: \tilde{M} \rightarrow \Delta^{n} \times I$, and an f.p. bounded sdr $\tilde{f}: \tilde{M} \rightarrow \mathbf{R}^{m} \times\{0\} \times F \times[0,1] \times$ $\Delta^{n} \times I$ which gives rise to a homotopy in $\mathscr{W} h\left(\mathbf{R}^{m} \times F \times[0,1]\right)$ showing that $\beta([h])=\beta\left(\left[h^{\prime}\right]\right)$.

PROPOSITION 6.3. $\beta$ is a group homomorphism. 
Proof. This follows almost immediately from the definitions.

The following simple variation of Alexander's trick will be useful in the next proposition.

LEMMA 6.4. Let $h: \mathbf{R}^{m+1} \times F \times[0,1] \times \Delta^{n} \rightarrow \mathbf{R}^{m+1} \times F \times[0,1] \times \Delta^{n}$ be a bounded homeomorphism such that $h=\mathrm{id}$ on $\left(\mathbf{R}^{m} \times(-\infty, 0] \times F \times[0,1] \times \Delta^{n}\right) \cup\left(\mathbf{R}^{m+1} \times F\right.$ $\left.\times\{0\} \times \Delta^{n}\right) \cup\left(\mathbf{R}^{m+1} \times F \times[0,1] \times \partial \Delta^{n}\right)$. Then $h$ is $f . p$. boundedly isotopic to the identity $\operatorname{rel}\left(\mathbf{R}^{m} \times(-\infty, 0] \times F \times[0,1] \times \Delta^{n}\right) \cup\left(\mathbf{R}^{m+1} \times F \times\{0\} \times \Delta^{n}\right) \cup\left(\mathbf{R}^{m+1} \times\right.$ $\left.F \times[0,1] \times \partial \Delta^{n}\right)$.

Proof. Define $\Theta_{s}: \mathbf{R} \rightarrow \mathbf{R}$ for $0 \leqslant s<1$ by $\Theta_{s}(t)=t-s /(s-1)$. Then $\Theta_{s}$ induces $\tilde{\Theta}_{s}: \mathbf{R}^{m+1} \times F \times[0,1] \times \Delta^{n} \rightarrow \mathbf{R}^{m+1} \times F \times[0,1] \times \Delta^{n}$ defined by $\tilde{\Theta}_{s}=\mathrm{id}$ $\times \Theta_{s} \times$ id. Define $h_{s}: \mathbf{R}^{m+1} \times F \times[0,1] \times \Delta^{n} \rightarrow \mathbf{R}^{m+1} \times F \times[0,1] \times \Delta^{n}$ by $h_{s}=$ $\tilde{\Theta}_{s}^{-1} h_{s} \tilde{\Theta}_{s}$ for $0 \leqslant s<1$ and $h_{1}=\mathrm{id}$. Then $h_{s}: h \simeq \mathrm{id}, 0 \leqslant s \leqslant 1$, is the desired isotopy.

Proposition 6.5. $\beta$ is injective.

Proof. Let $[h] \in \pi_{n} \mathscr{C}_{b}\left(\mathbf{R}^{m+1} \times F\right)$ such that $\beta([h])=0$. Suppose $\beta([h])=$ $k_{*}(\tau(f))$ is defined as above. Thus, we have an f.p. bounded sdr $f: M \rightarrow \mathbf{R}^{m} \times\{0\}$ $\times F \times[0,1] \times \Delta^{n}$ such that $\tau(f)=0$.

As a representative of 0 in $\pi_{n} \mathscr{W} h\left(\mathbf{R}^{m} \times F \times[0,1]\right)$ we use the projection pr: $\mathbf{R}^{m} \times[0, L] \times F \times[0,1] \times \Delta^{n} \rightarrow \mathbf{R}^{m} \times F \times[0,1] \times \Delta^{n}$. By Proposition 3.6 there is an f.p. homeomorphism $H: M \rightarrow \mathbf{R}^{m} \times[0, L] \times F \times[0,1] \times \Delta^{n}$ such that pr $H$ is boundedly homotopic to $f$ rel $\rho^{-1}\left(\partial \Delta^{n}\right)$ and $H \mid \mathbf{R}^{m} \times\{0\} \times F \times[0,1] \times \Delta^{n}=$ id. (This latter statement is somewhat stronger than Proposition 3.6. We are actually using the fact that $f$ is a sdr, the definition of torsion, and Proposition 3.4.) By sliced Z-set unknotting we may also assume that $H \mid\left(\mathbf{R}^{m} \times[0, L] \times F \times\{0\} \times \Delta^{n}\right) \cup\left(\mathbf{R}^{m} \times\right.$ $\left.[0, L] \times F \times[0,1] \times \partial \Delta^{n}\right) \cup\left(\mathbf{R}^{m} \times\{L\} \times F \times[0,1] \times \Delta^{n}\right)$ is the identity. The homotopies needed to apply sliced $Z$-set unknotting come from pushing along the $[0, L]$ and $[0,1]$ factors.

Now define a hybrid concordance $\tilde{h}: \mathbf{R}^{m+1} \times F \times[0,1] \times \Delta^{n} \rightarrow \mathbf{R}^{m+1} \times F \times$ $[0,1] \times \Delta^{n}$ by

$$
\tilde{h}= \begin{cases}h & \text { on } \mathbf{R}^{m} \times[L,+\infty) \times F \times[0,1] \times \Delta^{n}, \\ H^{-1} & \text { on } \mathbf{R}^{m} \times[0, L] \times F \times[0,1] \times \Delta^{n}, \\ \text { id } & \text { on } \mathbf{R}^{m} \times(-\infty, 0] \times F \times[0,1] \times \Delta^{n} .\end{cases}
$$

Apply Lemma 6.4 to see that $h^{-1} \tilde{h}$ and $\tilde{h}$ are both f.p. boundedly isotopic to the identity $\operatorname{rel}\left(\mathbf{R}^{m+1} \times F \times\{0\} \times \Delta^{n}\right) \cup\left(\mathbf{R}^{m+1} \times F \times[0,1] \times \partial \Delta^{n}\right)$. This shows that $[h]=[\tilde{h}]=[\mathrm{id}]$ in $\pi_{n} \mathscr{C}_{b}\left(\mathbf{R}^{m+1} \times F\right)$. Thus, $[h]=0$ and the kernel of $\beta$ is 0 .

Proposition 6.6. $\beta$ is surjective.

Proof. It will be shown that $k_{*}^{-1} \beta: \pi_{n} \mathscr{C}_{b}\left(\mathbf{R}^{m+1} \times F\right) \rightarrow \pi_{n} \mathscr{W} h\left(\mathbf{R}^{m} \times F \times[0,1]\right)$ is surjective. We treat the two cases $n=0$ and $n \geqslant 1$ separately. For the $n=0$ case let $\tau \in \pi_{0} \mathscr{W} h\left(\mathbf{R}^{m} \times F \times[0,1]\right)$. Using the method of [11, p. 200] we will construct a bounded concordance $h: \mathbf{R}^{m+1} \times F \times[0,1] \rightarrow \mathbf{R}^{m+1} \times F \times[0,1]$ such that $k_{*}^{-1} \beta([h])=\tau$. 
We first need a decomposition $M_{i} \cup N_{i}=\mathbf{R}^{m} \times[i, i+1] \times F \times[0,1]$ for every integer $i$, where

(i) $M_{i}$ and $N_{i}$ are $Q$-manifolds,

(ii) $M_{i} \cap N_{i}=P_{i}$ is a $Q$-manifold collared in both $M_{i}$ and $N_{i}$,

(iii) $\mathbf{R}^{m} \times\{i\} \times F \times[0,1] \subset$ int $M_{i}$ and $\mathbf{R}^{m} \times\{i+1\} \times F \times[0,1] \subset$ int $N_{i}$,

(iv) $M_{i} \cap\left(\mathbf{R}^{m} \times[i, i+1] \times F \times\{0\}\right)=\mathbf{R}^{m} \times\left[i, i+\frac{1}{2}\right] \times F \times\{0\}$ and $N_{i} \cap\left(\mathbf{R}^{m}\right.$ $\times[i, i+1] \times F \times\{0\})=\mathbf{R}^{m} \times\left[i+\frac{1}{2}, i+1\right] \times F \times\{0\}$,

(v) the projections $M_{i} \rightarrow \mathbf{R}^{m} \times\{i\} \times F \times[0,1]$ and $N_{i} \rightarrow \mathbf{R}^{m} \times\{i+1\} \times F \times$ $[0,1]$ are bounded sdr's with torsions $\tau$ and $-\tau$, respectively,

(vi) there is a homeomorphism $g_{i}: \mathbf{R}^{m} \times F \times[0,1] \rightarrow P_{i}$ such that $g_{i}$ is boundedly homotopic in $M_{i}$ to the inclusion $\mathbf{R}^{m} \times F \times[0,1] \rightarrow \mathbf{R}^{m} \times\{i\} \times F \times[0,1]$, and in $N_{i}$ to the inclusion $\mathbf{R}^{m} \times F \times[0,1] \rightarrow \mathbf{R}^{m} \times\{i+1\} \times F \times[0,1]$. Also, $g_{i} \mid: \mathbf{R}^{m} \times F$ $\times\{0\} \rightarrow \mathbf{R}^{m} \times\left\{\frac{1}{2}\right\} \times F \times\{0\}$ is the identity.

To get such a decomposition (say for $i=0$ ), let $\mathbf{R}^{m} \times\left[0, \frac{1}{2}\right] \times F \times[0,1]$ and $\mathbf{R}^{m} \times\left[\frac{1}{2}, 1\right] \times F \times[0,1]$ be contained as $Z$-sets in the $Q$-manifolds $M$ and $N$, respectively. Let $f_{0}: M \rightarrow \mathbf{R}^{m} \times\left[0, \frac{1}{2}\right] \times F \times[0,1]$ and $f_{1}: N \rightarrow \mathbf{R}^{m} \times\left[\frac{1}{2}, 1\right] \times F \times$ $[0,1]$ be bounded sdr's such that $f_{j}$ followed by projection to $\mathbf{R}^{m} \times\{j\} \times F \times[0,1]$ for $j=0,1$ represents $\tau$ or $-\tau$, respectively. Let $\tilde{M}$ be the union of $M$ and $N$ along $\mathbf{R}^{m} \times\left\{\frac{1}{2}\right\} \times F \times[0,1]$ and define $\tilde{f}: \tilde{M} \rightarrow \mathbf{R}^{m} \times\{0\} \times F \times[0,1]$ to be $f_{0} \cup f_{1}$ followed by projection. Then $\tau(\tilde{f})=0$.

Therefore, one can construct a homeomorphism $H: \tilde{M} \rightarrow \mathbf{R}^{m} \times[0,1] \times F \times[0,1]$ such that $H \mid\left(\mathbf{R}^{m} \times\{0,1\} \times F \times[0,1]\right) \cup\left(\mathbf{R}^{m} \times[0,1] \times F \times\{0\}\right)=$ id and $H$ followed by projection is boundedly homotopic to $\tilde{f}$. Then set $M_{0}=H(M)$ and $N_{0}=H(N)$.

Now one defines a bounded homeomorphism $h_{i}: \mathbf{R}^{m} \times\left[i-\frac{1}{2}, i+\frac{1}{2}\right] \times F \times[0,1]$ $\rightarrow N_{i-1} \cup M_{i}$ so that $h_{i} \mid \mathbf{R}^{m} \times\left[i-\frac{1}{2}, i+\frac{1}{2}\right] \times F \times\{0\}=$ id. By using $Z$-set unknotting we can assume $h_{i}\left|\mathbf{R}^{m} \times\left\{i+\frac{1}{2}\right\} \times F \times[0,1]=h_{i+1}\right|$. Then the $h_{i}$ 's piece together to define $h$.

For the case $n \geqslant 1$ let $\tau \in \pi_{n} \mathscr{W h}\left(\mathbf{R}^{m} \times F \times[0,1]\right)$ be given. Let $\alpha(\tau)=[h] \in$ $\pi_{n-1} \mathscr{C}_{b}\left(\mathbf{R}^{m} \times F \times[0,1]\right)$, where $h: \mathbf{R}^{m} \times F \times[0,1] \times[0,1] \times \Delta^{n-1} \rightarrow \mathbf{R}^{m} \times F \times$ $[0,1] \times[0,1] \times \Delta^{n-1}$ is a bounded homeomorphism f.p. over $\Delta^{n-1}$ such that $h \mid \mathbf{R}^{m} \times$ $F \times[0,1] \times\{0\} \times \Delta^{n-1}=$ id and $h \mid \mathbf{R}^{m} \times F \times[0,1] \times[0,1] \times \partial \Delta^{n-1}=$ id. By sliced $Z$-set unknotting we may assume that $h \mid \mathbf{R}^{m} \times F \times\{0,1\} \times[0,1] \times \Delta^{n-1}=$ id.

By a familiar construction (see [1, 20, 21 and 22]), we will show there is an element $[\tilde{h}] \in \pi_{n} \mathscr{C}_{b}\left(\mathbf{R}^{m+1} \times F\right)$ such that $\alpha k_{*}^{-1} \beta([\tilde{h}])=[h]$. Since $\alpha$ is injective, we will have $k_{*}^{-1} \beta([\tilde{h}])=\tau$.

To construct $\tilde{h}$, let $\sigma:(0,1) \rightarrow \mathbf{R}$ be an increasing homeomorphism such that $\boldsymbol{\sigma}\left(\frac{1}{2}\right)=0$. For $0<u<1$, define $T_{u}: \mathbf{R}^{m} \times F \times \mathbf{R} \times[0,1] \times \Delta^{n-1} \rightarrow \mathbf{R}^{m} \times F \times \mathbf{R} \times$ $[0,1] \times \Delta^{n-1}$ by $T_{u}(x, f, y, s, t)=(x, f, y+\sigma(u), s, t)$. Define $\bar{h}: \mathbf{R}^{m} \times F \times \mathbf{R} \times$ $[0,1] \times \Delta^{n-1} \rightarrow \mathbf{R}^{m} \times F \times \mathbf{R} \times[0,1] \times \Delta^{n-1}$ by extending $h$ via the identity. Recall $I=[0,1]$. Now define $\tilde{h}: \mathbf{R}^{m} \times F \times \mathbf{R} \times[0,1] \times \Delta^{n-1} \times I \rightarrow \mathbf{R}^{m} \times F \times \mathbf{R} \times[0,1]$ $\times \Delta^{n-1} \times I$ by

$$
\tilde{h}(x, f, y, s, t, u)= \begin{cases}\left(T_{u}^{-1} h T_{u}(x, f, y, s, t), u\right) & \text { for } 0<u<1 \\ (x, f, y, s, t, u) & \text { for } u=0,1\end{cases}
$$


Identify $\Delta^{n-1} \times I$ with $\Delta^{n}$ in such a way that $\Delta^{n-1} \times\{1\}$ is identified with $J^{n-1}$. Then $[\tilde{h}] \in \pi_{n} \mathscr{C}_{b}\left(\mathbf{R}^{m+1} \times F\right)$ and we claim $\alpha k_{*}^{-1} \beta([\tilde{h}])=[h]$. First recall how $k_{*}^{-1} \beta([\tilde{h}])$ is defined. Choose $L>0$ large and set

$$
M=\tilde{h}\left(\mathbf{R}^{m} \times F \times(-\infty, L] \times[0,1] \times \Delta^{n-1} \times I\right) \backslash\left(\mathbf{R}^{m} \times F \times(-\infty, 0) \times[0,1] \times \Delta^{n-1} \times I\right) .
$$

Maps $\rho: M \rightarrow \Delta^{n-1} \times I$ and $f: M \rightarrow \mathbf{R}^{m} \times F \times\{0\} \times[0,1] \times \Delta^{n-1} \times I$ are defined to be the restrictions of the projections. Then $k_{*}^{-1} \beta([\tilde{h}])=\tau(f)$.

To define $\alpha(\tau(f))$ we need a trivializing homeomorphism $H$ for $\rho: M \rightarrow \Delta^{n-1} \times I$. Define $H: \mathbf{R}^{m} \times F \times[0, L] \times[0,1] \times \Delta^{n-1} \times I \rightarrow M$ by

$$
H(x, f, y, s, t, u)= \begin{cases}(\bar{h}(x, f, y, s, t), u) & \text { for } \frac{1}{2} \leqslant u \leqslant 1, \\ \left(T_{u}^{-1} \bar{h} T_{u}(x, f, y, s, t), u\right) & \text { for } 0<u<\frac{1}{2}, \\ (x, f, y, s, t, u) & \text { for } u=0 .\end{cases}
$$

Note that $p f H$ is boundedly close to projection.

It follows that $\alpha(\tau(f))$ is represented by $H \mid \mathbf{R}^{m} \times F \times[0, L] \times[0,1] \times \Delta^{n-1} \times\{1\}$ (after $[0, L]$ is identified with $[0,1])$. This is clearly the class of $h$. Thus $\alpha k_{*}^{-1} \beta([\tilde{h}])=$ $[h]$ as desired.

\section{REFERENCES}

1. D. R. Anderson and W.-C. Hsiang, The functors $K_{-i}$ and pseudoisotopies of polyhedra, Ann. of Math (2) 105 (1977), 201-223.

2. H. Bass, Algebraic K-theory, Benjamin, New York, 1968.

3. D. W. Carter, Localization in lower algebraic K-theory, Comm. Algebra 8 (1980), 603-622.

4. Lower K-theory of nilpotent groups, J. Algebra 66 (1980), 134-146.

5. __ Lower K-theory of finite groups, Comm. Algebra 8 (1980), 1927-1937.

6. T. A. Chapman, Lectures on Hilbert cube manifolds, C.B.M.S. Regional Conf. Ser. in Math., no. 28, Amer. Math. Soc., Providence, R.I., 1976.

7. __ Approximating maps into fiber bundles by homeomorphisms, Rocky Mountain J. Math. 10 (1980), 333-350.

8. Approximation results in Hilbert cube manifolds, Trans. Amer. Math. Soc. 262 (1980), $303-334$.

9. Approximation results in topological manifolds, Mem. Amer. Math. Soc. No. 34 (1981).

10. __ Proper fibrations with Hilbert cube manifold fibers, Topology Appl. 12 (1981), 19-33.

11. T. A. Chapman and L. C. Siebenmann, Finding a boundary for a Hilbert cube manifold, Acta Math. 137 (1976), 171-208.

12. T. A. Chapman and R. Y. T. Wong, On homeomorphisms of infinite dimensional bundles. III, Trans. Amer. Math. Soc. 191 (1974), 269-276.

13. M. M. Cohen, $A$ course in simple-homotopy theory, Springer-Verlag, New York, 1970.

14. D. S. Coram and P. F. Duvall, Jr., Approximate fibrations, Rocky Mountain J. Math. 7 (1977), 275-288.

15. , Approximate fibrations and a moveability condition for maps, Pacific J. Math. 72 (1977), $41-56$

16. A. Dold, Partitions of unity in the theory of fibrations, Ann. of Math. (2) 78 (1963), 223-225.

17. A. Fathi and Y. M. Visetti, Deformation of open embeddings of Q-manifolds, Trans. Amer. Math. Soc. 224 (1976), 427-436.

18. S. Ferry, The homeomorphism group of a compact Hilbert cube manifold is an ANR, Ann. of Math. (2) 106 (1977), 101-119.

19. S. M. Gersten, On the spectrum of algebraic K-theory, Bull. Amer. Math. Soc. 78 (1972), 216-219.

20. A. E. Hatcher, Higher simple homotopy theory, Ann. of Math. (2) 102 (1975), 101-138.

21. Concordance spaces, higher simple-homotopy theory, and applications, Proc. Sympos. Pure Math., vol. 32, Amer. Math. Soc., Providence, R.I., 1978, pp. 3-21. 
22. W.-C. Hsiang and R. W. Sharpe, Parametrized surgery and isotopy, Pacific J. Math. 67 (1976), 401-459.

23. C. B. Hughes, Spaces of approximate fibrations on Hilbert cube manifolds, preprint.

24. Approximate fibrations and bundle maps on Hilbert cube manifolds, Topology Appl. 15 (1983), 159-172.

25. R. C. Kirby and L.C. Siebenmann, Foundational essays on topological manifolds, Princeton Univ. Press, Princeton, N.J., 1977.

26. J. P. May, Simplicial objects in algebraic topology, Van Nostrand-Reinhold, Princeton, N.J., 1967.

27. F. Quinn, Ends of maps. I, Ann. of Math. (2) 110 (1979), 275-331.

28. __ Ends of maps. II, Invent. Math. 68 (1982), 353-424.

29. L. C. Siebenmann, The obstruction to finding a boundary for an open manifold of dimension greater than five, Thesis, Princeton University, 1965.

30. __ Infinite simple homotopy types, Indag. Math. 32 (1970), 479-495.

31. Deformation of homeomorphism on stratified sets, Comment. Math. Helv. 47 (1972), $123-163$.

32. E. H. Spanier, Algebraic topology, McGraw-Hill, New York, 1966.

Department of Mathematics, University of Utah, Salt Lake City, Utah 84112 